\title{
Impact of early cART in the gut during acute HIV infection
}

Claire Deleage, ${ }^{1}$ Alexandra Schuetz, ${ }^{2,3}$ W. Gregory Alvord, ${ }^{4}$ Leslie Johnston, ${ }^{1}$ Xing-Pei Hao, ${ }^{5}$ David R. Morcock, ${ }^{1}$ Rungsun Rerknimitr, ${ }^{6}$ James L.K. Fletcher, ${ }^{7,8}$ Suwanna Puttamaswin, ${ }^{7,8}$ Nittaya Phanuphak, ${ }^{7,8}$ Robin Dewar, ${ }^{9}$ Joseph M. McCune, ${ }^{10}$ Irini Sereti, ${ }^{11}$ Merlin Robb, ${ }^{3}$ Jerome H. Kim, ${ }^{3,7,12}$ Timothy W. Schacker, ${ }^{13}$ Peter Hunt, ${ }^{14}$ Jeffrey D. Lifson, ${ }^{1}$ Jintanat Ananworanich, ${ }^{3,7}$ and Jacob D. Estes ${ }^{1}$ on behalf of the RV254/SEARCH 010 and RV304/SEARCH 013 Study Groups ${ }^{15}$

'AIDS and Cancer Virus Program, Frederick National Laboratory for Cancer Research, Leidos Biomedical Research Inc., Frederick, Maryland, USA. 'Department of Retrovirology, Armed Forces Research Institute of Medical Sciences, Bangkok, Thailand. ${ }^{3}$ US Military HIV Research Program, Henry M. Jackson Foundation for the Advancement of Military Medicine, Bethesda, Maryland, USA. ${ }^{4}$ Statistical Consulting, Data Management Services Inc., Frederick, Maryland, USA. ${ }^{5}$ Pathology and Histotechnology Laboratory, Frederick National Laboratory for Cancer Research, Leidos Biomedical Research Inc., Frederick, Maryland, USA. ${ }^{6}$ Chulalongkorn University, Bangkok, Thailand. ${ }^{7}$ SEARCH, Bangkok, Thailand. ${ }^{8}$ Thai Red Cross AIDS Research Centre, Bangkok, Thailand. ${ }^{9}$ Virus Isolation and Serology Laboratory, Applied and Developmental Research Directorate, Science Applications International Corp., Frederick Inc. National Cancer Institute, Frederick Cancer Research and Development Center, Frederick, Maryland, USA. ${ }^{10}$ Division of Experimental Medicine, Department of Medicine, UCSF, San Francisco, California, USA. "National Institute of Allergy and Infectious Diseases, NIH, Bethesda, Maryland, USA. ${ }^{12}$ International Vaccine Institute, Seoul, South Korea. ${ }^{13}$ Department of Medicine, University of Minnesota, Minneapolis, Minnesota, USA. ${ }^{14}$ Positive Health Program, Department of Medicine, UCSF, San Francisco, California, USA. ${ }^{15}$ The RV254/ SEARCH 010 and RV304/SEARCH 013 Study Groups are detailed in the Supplemental Acknowledgments.

Early after HIV infection there is substantial depletion of CD4+ $\mathrm{T}$ cells in the gastrointestinal (GI) tract lamina propria (LP), with associated epithelial barrier damage, leading to microbial translocation and systemic inflammation and immune activation. In this study, we analyzed these early events in the $\mathrm{GI}$ tract in a cohort of Thai acute HIV-infected patients and determined the effect of early combination antiretroviral treatment (CART). HIV-uninfected and chronically and acutely HIV-infected patients at different Fiebig stages (I-V) underwent colonic biopsies and then received CART. Immunohistochemistry and quantitative image analysis were performed on crosssectional and longitudinal colon biopsy specimens (day 0 to week 96 ) to measure $\mathrm{Gl}$ tract damage (infiltration of polymorphonuclear cells), inflammation (Mx1, TNF- $\alpha$ ), immune activation (Ki-67), and the $\mathrm{CD}^{+} \mathrm{T}$ cell population in the LP. The magnitude of $\mathrm{Gl}$ tract damage, immune activation, and inflammation was significantly increased, with significantly depleted CD4+ $T$ cells in the LP in all acutely infected groups prior to CART compared with HIV-uninfected control participants. While most patients treated during acute infection resolved $\mathrm{CI}$ tract inflammation and immune activation back to baseline levels after 24 weeks of cART, most acutely infected participants did not restore their $\mathrm{CD4}^{+} \mathrm{T}$ cells after 96 weeks of cART.

Conflict of interest: J. Ananworanich has received honorarium from ViiV Healthcare, Merck, and Tetralogic for her participation in advisory meetings.

Submitted: February 12, 2016 Accepted: May 19, 2016 Published: July 7, 2016

Reference information: JCI Insight. 2016;1(10):e87065. doi:10.1172/jci.insight.87065.

\section{Introduction}

The number of HIV-infected individuals receiving combination antiretroviral therapy (cART) has tripled in the last 5 years (1). Initiation of cART typically leads to a rapid and significant reduction in viral replication to the extent that the plasma HIV viral load becomes undetectable and the peripheral blood CD4 ${ }^{+}$ $\mathrm{T}$ cell count increases in the majority of those who receive treatment. Nonetheless, HIV-infected individuals, particularly those who start cART at later stages of disease, are still afflicted with "non-AIDS" comorbidities and diseases, with a shorter life expectancy than age-matched, uninfected controls (2-5). It is hypothesized that persistent gastrointestinal (GI) tract damage despite cART leads to recurrent microbial translocation and contributes to sustained inflammation and immune activation, which are strongly associated with non-AIDS coinfections and comorbidities (6-9). Furthermore, recent data suggest that long-term cART does not readily reverse the extensive fibrotic damage to lymphoid tissues that is evident 
in HIV-infected individuals prior to cART initiation, particularly when treatment is started in the chronic phase of infection (10). Many of the non-AIDS morbidities are correlated with persistently elevated levels of immune activation and inflammation during HIV infection (11). Even though cART significantly decreases most of markers of immune activation and inflammation measurable in the blood, many studies have reported ongoing $\mathrm{T}$ cell activation and inflammation in tissues of patients undergoing long-term cART, which could be a result of persistent dysfunction and damage in the GI tract $(6,12,13)$.

The GI tract is an organ system that is particularly affected by HIV infection. Substantial CD4 ${ }^{+} \mathrm{T}$ cell depletion in the GI tract occurs early during acute HIV infection (AHI) $(14,15)$, and, while some studies demonstrated partial recovery of $\mathrm{CD}^{+} \mathrm{T}$ cells following cART, these cells typically fail to be restored in the lamina propria (LP), the immune effector site of the GI tract (16). The reasons for the lack of reconstitution of $\mathrm{CD}^{+} \mathrm{T}$ cells in the LP of the GI tract are incompletely understood, but persistent GI tract damage and inflammation; loss of $\mathrm{CD}^{+}$central memory $\mathrm{T}$ cells, resulting in reduced production of $\mathrm{CD}^{+}$effector memory $\mathrm{T}$ cells; and residual ongoing HIV replication, despite the significant benefits of cART, may play a role $(17,18)$. While most studies have investigated the effects of cART in chronically HIV-infected (CHI) individuals, several studies have examined the potential benefits of administering cART during acute infection, finding that such an intervention is associated with the preservation immune function (19), limitation of the size of reservoir $(20,21)$, protection of long-lived cells from persistent infection (22), and enhanced recovery of $\mathrm{CD}^{+} \mathrm{T}$ cell numbers and function in blood (23, 24) and in the GI tract (25). However, it remains unclear whether early cART will prevent the loss of or restore $\mathrm{CD}^{+} \mathrm{T}$ cells within the LP effector site of the GI tract and limit or reverse the immunopathology associated with HIV-1 infection within the GI tract.

To examine these events more closely, we studied GI tract samples collected from the RV254/SEARCH 010 cohort in Thailand. Participants in this cohort were identified during early acute HIV-1 infection and immediately offered cART; they were also willing to undergo colonic biopsies $(26,27)$. This cohort represents a unique opportunity to investigate the effect of cART on the GI tract at the earliest time points clinically feasible after initial HIV infection. In this study, we compared the effects of cART initiated during acute or chronic phases of infection, specifically focusing on the immunopathology of the GI tract. We analyzed the effect of the timing of cART initiation on GI tract damage, the CD4 ${ }^{+} \mathrm{T}$ cell population size within the LP, and different aspects of immune activation and inflammation before and after 24 and 96 weeks of treatment.

\section{Results}

Clinical characteristics and virological response to cART in plasma and colonic tissue. We identified 41 individuals with AHI (Table 1), staged according to Fiebig classification (28), who were willing to undergo colonic biopsy. Eighteen participants were identified by pooled nucleic acid test (NAT) (nonreactive HIV IgM antibody) and classified as Fiebig I/II (FI/II), and 23 were identified by sequential enzyme immunoassay (EIA) (reactive HIV IgM/non-reactive HIV IgG antibody) and classified as Fiebig III-V (FIII+) (28). Additionally, 10 age-, gender-, and risk group-matched HIV-uninfected (HIV-) individuals from Thailand served as negative controls, and $16 \mathrm{CHI}$ individuals (6 of which were from Thailand and received treatment in the chronic phase of the infection and 10 of which were from the US and were untreated) were included to serve as positive controls. Participants were mainly young men who have sex with men (73\%) infected with HIV-1 CRF01_AE (30 of 41), with a median time since history of HIV-1 exposure of 15 days (range 4-32), a median CD4 ${ }^{+} \mathrm{T}$ cell count of 466 cells $/ \mathrm{mm}^{3}$ (Figure 1A), a median plasma HIV RNA load of $5.51 \log _{10}$ copies/ml (Figure 1B), and a median colon HIV RNA load of $2.85 \log _{10}$ copies/ mg tissue (Figure 1C). The plasma as well as the colonic HIV RNA load increased significantly with progression of infection from FI/II to treatment (plasma HIV RNA: FI/II $4.97 \log _{10}$ copies/ml vs. FIII+ 5.63 $\log _{10}$ copies/ml; $P=0.002$; colonic HIV RNA: FI/II $1.98 \log _{10}$ copies/mg tissue vs. treatment $3.12 \log _{10}$ copies/mg tissue; $P=0.01$ ) (Figure $1, \mathrm{~B}$ and $\mathrm{C}$ ).

We determined the anatomical localization of productively infected HIV viral $\mathrm{RNA}^{+}\left(\mathrm{vRNA}^{+}\right)$cells by performing standard in situ hybridization using lineage-specific riboprobes to either HIV-1 CRF01_AE or HIV-1 clade B. Many patients with AHI FI/II had abundant vRNA cells in both the LP and lymphoid aggregates (Figure 1D), highlighting the early detection of these individuals. By contrast, in the later FIII+ acute phase, HIV-1 vRNA ${ }^{+}$cells were absent from the LP and restricted to the lymphoid aggregates (Figure 1D), consistent with rapid depletion of $\mathrm{CD}^{+} \mathrm{T}$ cells within the LP. 
Table 1. Clinical baseline characteristics and demographics of study participants

\begin{tabular}{|c|c|c|c|c|c|}
\hline Characteristics & $\begin{array}{l}\text { Acute HIV infected } \\
\text { FI/II }(n=19)\end{array}$ & $\begin{array}{l}\text { Acute HIV infected FIII+ } \\
\qquad(n=22)\end{array}$ & $\begin{array}{l}\text { Chronic untreated } \\
\text { American HIV infected } \\
\qquad(n=10)\end{array}$ & $\begin{array}{l}\text { Chronic treated Thai } \\
\text { HIV infected }(n=6)\end{array}$ & $\begin{array}{l}\text { HIV uninfected } \\
\qquad(n=10)\end{array}$ \\
\hline Median age (yr) & 31 & 25 & $43(24-61)$ & $26(19-31)^{A}$ & $31(23-41)^{A}$ \\
\hline \multicolumn{6}{|l|}{ Risk behavior, $n$ (\%) } \\
\hline MSM & 15 & 18 & - & $6(100 \%)$ & NA \\
\hline I & 10 & NA & NA & & NA \\
\hline II & 9 & NA & & & \\
\hline III & NA & 19 & & & \\
\hline V & NA & 2 & NA & & NA \\
\hline VI & NA & 1 & NA & & NA \\
\hline $\begin{array}{r}\left(\log _{10} \text { copies } / \mathrm{ml}\right) \\
\text { Plasma do } \\
\text { Colonic do }\end{array}$ & 1.98 & 3.12 & NA & NA & \\
\hline Median CD4 count (cell/mm³) & & & $480(25,988)$ & $395.5(286,883)^{A}$ & NA \\
\hline do & $538(269,1127)^{A}$ & $386(132,740)^{A}$ & & & \\
\hline 24 weeks & $600(291,1145)$ & $571(301,908)$ & & & \\
\hline 96 weeks & $634(451,1154)$ & $592(378,1111)$ & & & \\
\hline HIV subtype by MHAbce ${ }^{B}$ & $3 \mathrm{NT}+1 \mathrm{ND}$ & $3 N T+2 N D$ & & & \\
\hline CRF01_AE & 14 & 17 & ND & ND & NA \\
\hline Recombinant $A E / B$ & 1 & - & ND & ND & NA \\
\hline $\begin{array}{l}\text { AMinimum value, maximum valu } \\
\text { CRF01_AE/B, six were nontypabl } \\
\text { Fiebig I, positive HIV RNA, negat } \\
\text { EIA; Fiebig III, positive HIV RNA, } \\
\text { negative p24 antigen, positive th } \\
\text { EIA, positive Western blot excep }\end{array}$ & $\begin{array}{l}\text { BMHAbce, multiregion h } \\
\text { (NT) and three were not } \\
\text { e p24 antigen, negative t } \\
\text { ositive p24 antigen, posit } \\
\text { d generation EIA, indeter } \\
\text { 31; NA, not applicable; S }\end{array}$ & $\begin{array}{l}\text { ybridization assay distingu } \\
\text { determined by the time we } \\
\text { third generation EIA; Fiebig } \\
\text { tive third generation EIA, ne } \\
\text { rminate Western blot; Fiebi } \\
\mathrm{STI} \text {, sexually transmitted inf }\end{array}$ & $\begin{array}{l}\text { hing between subtypes } \\
\text { vere writing the manuscr } \\
\text {, positive HIV RNA, posi } \\
\text { gative Western blot; Fieb } \\
\text { V, positive HIV RNA, pos } \\
\text { ction; HBV, hepatitis B v }\end{array}$ & $\begin{array}{l}\text { C and CRF01_AE. One p } \\
\text { t; MSM, men who have } \\
\text { e p24 antigen, negative } \\
\text { IV, positive HIV RNA, p } \\
\text { ive p24 antigen, positiv }\end{array}$ & $\begin{array}{l}\text { articipant was } \\
\text { sex with men; } \\
\text { third generation } \\
\text { sitive or } \\
\text { third generation }\end{array}$ \\
\hline
\end{tabular}

After the initiation of cART, the median peripheral blood CD4 ${ }^{+} \mathrm{T}$ cell count increased to 591 cells/ $\mathrm{mm}^{3}$ at week 24 and to 617 cells $/ \mathrm{mm}^{3}$ at week 96 for all groups (Figure 1E). Plasma HIV-1 RNA decreased below 30 copies $/ \mathrm{ml}$, reaching an undetectable level in 98\% (40 of 41) of patients tested at week 24 and $95 \%$ (39 of 41) at week 96 (Figure 1F). By 24 weeks of cART, HIV-1 RNA levels in gut tissue were below 50 copies/mg tissue in $90 \%$ (28 of 31) of the participants tested (Figure $1 \mathrm{G}$ ). After only 24 weeks of treatment, we were not able to detect any vRNA ${ }^{+}$cells by standard in situ hybridization in colonic biopsies from any of the AHI or CHI participants placed on cART during AHI or CHI (data not shown).

GI tract epithelial barrier integrity, early GI tract damage, inflammation/immune activation, and CD4 $T$ cell depletion during AHI. We sought to determine whether participants identified after AHI would have evidence for $\mathrm{CD}^{+} \mathrm{T}$ cell depletion in the LP of the GI tract. During AHI, there was a significant decrease in $\mathrm{CD}^{+} \mathrm{T}$ cells within the LP in both FI/II and FIII+ patients compared with HIV- individuals (percentage area $\mathrm{CD}^{+} \mathrm{T}$ cells in LP, FI/II: $1.35 \%$ and FIII+: $0.62 \%$ vs. HIV ${ }^{-}: 2.14 \% ; P=0.0076$ and $P<0.0001$, respectively) (Figure 2), consistent with our previous observations (25). The FIII+ group had more severe depletion of $\mathrm{CD}^{+} \mathrm{T}$ cells; it was significantly lower than that found in the FI/II group at baseline (percentage area CD4 ${ }^{+} \mathrm{T}$ cells in LP, FI/II: $1.35 \%$ vs. FIII+: $0.62 \% ; P=0.005$ ). As expected, the CD4 ${ }^{+} \mathrm{T}$ cell population size was significantly decreased in untreated patients with $\mathrm{CHI}$ compared with $\mathrm{HIV}^{-}$individuals (percentage area CD4 ${ }^{+} \mathrm{T}$ cells in LP CHI no cART: $0.894 \%$ vs. HIV $: 2.14 \% ; P=0.001$ ) (Figure 2 ). In contrast to $\mathrm{CD}^{+} \mathrm{T}$ cells, myeloid lineage cell populations $\left(\mathrm{CD} 68^{+} / \mathrm{CD} 163^{+}\right)$were not significant different between any of the groups (data not shown), highlighting the specificity of the $\mathrm{CD} 4^{+} \mathrm{T}$ cell loss within the 

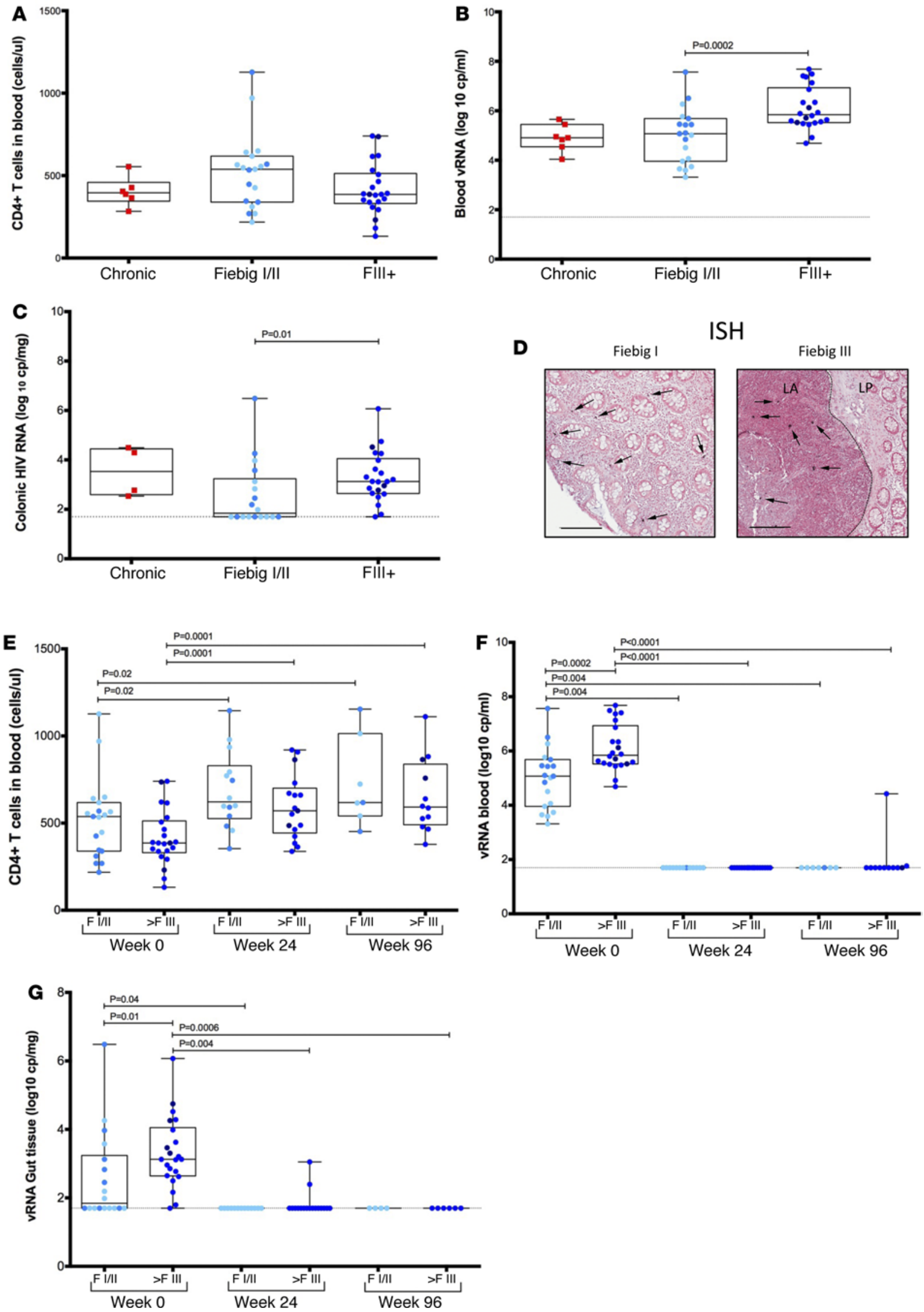
Figure 1. CD4 ${ }^{+} \mathrm{T}$ cell count and vRNA in blood and sigmoid biopsies. Thai acute patients presented no significant decrease of CD4 ${ }^{+} \mathrm{T}$ cell count in blood at the first visit compared with HIV chronic patients (A). Blood viral load (VL) was similar in Fiebig I/II (FI/II) ( $n=19)$ or Fiebig III-V (FIII+) $(n=22)$ to that observed in chronically infected individuals $(n=6)(B)$. Colonic viral RNA (vRNA) increased significantly with progression of infection from $\mathrm{FI} / \mathrm{II}$ to $\mathrm{FIII+}$ (C). Representative ISH shows infected cells transcribing vRNA in the laminal propria (LP) of an FI/II individual in both the LP and lymphoid aggregates (LA) of an FIII+ patient (D). The number of CD4+ T cells in the blood increased gradually after initiation of the combined antiretroviral therapy (CART) (E), while the vRNA in blood (F) and in sigmoid colon biopsies (G) decreased. (E-G) For Fl/Il group at week $0, n=19 ;$ week 24 , $n=15$; and week $96, n=8$. For FIII+ group at week $0, n=22$; week 24, $n=17$; and week $96 n=12$. Each dot represents the median of at least 3 biopsies per participant. Within the $\mathrm{FI} / \mathrm{II}$ group, lighter blue dots represent $\mathrm{FI}$ patients and darker blue represent the FII patients. Within FIII+ group, lighter blue dots represent FIII+ patients and darker dots represent FIV and FV patients. Scale bar: $100 \mu \mathrm{m}$. Following linear mixed-effects (Ime) analyses, post-hoc Ime contrasts and pairwise nonparametric (Wilcoxon) tests were performed. Benjamini-Hochberg adjusted $P$ values are reported. Data are presented as box-and-whisker plots with the lower and upper limits (bounds) of the box representing Q1 (25th percentile) and Q3 (75th percentile), respectively. The median (Q2, 50th percentile) is represented by the horizontal line within the box. Whiskers, delineated as horizontal stems, extend to the limits of the data. Data points (superimposed) are "jittered" to avoid overplotting.

LP of HIV-infected individuals. While the number of $\mathrm{CD} 8^{+} \mathrm{T}$ cells in the FI/II group was not significantly increased compared with the HIV- individuals (mean percentage area of $\mathrm{CD}^{+} \mathrm{T}$ cells in LP AHI, FI/II: $5.32 \%$ vs. HIV-: $4.69 \% ; P=0.2$ ) (data not shown), the FIII+ group showed a significant increase in $\mathrm{CD}^{+} \mathrm{T}$ cells compared with both FI/II patients and $\mathrm{HIV}^{-}$individuals (mean percentage area of $\mathrm{CD} 8^{+} \mathrm{T}$ cells in $\mathrm{LP}$ AHI, FIII+: $6.82 \%$ vs. FI/II: $5.32 \%$, HIV $: 2.14 \% ; P=0.09$ and $P=0.04$, respectively). However, the CD $8^{+}$ $\mathrm{T}$ cell level remained lower in both Fiebig groups compared with untreated participants with $\mathrm{CHI}$ (mean percentage area of $\mathrm{CD}^{+} \mathrm{T}$ cells in LP AHI, FI/II: $5.32 \%$, FIII+: $6.82 \%$ vs. CHI: $10.4 \% ; P=0.004$ and $P=0.02$, respectively). Collectively, these data highlight the dynamic and progressive nature of $\mathrm{CD} 4^{+} \mathrm{T}$ cell depletion and adaptive $\mathrm{T}$ cell responses in the LP of the GI tract during HIV infection.

Next, we sought to determine whether participants identified at early stages of HIV infection had limited GI tract epithelial damage and, as a result, limited microbial translocation. In a SIV-infected nonhuman primate (NHP) model, such damage is first evident late in the acute phase of infection (i.e., 14 dpi) (29). In humans, however, mucosal barrier integrity and microbial translocation are inherently difficult to evaluate and quantify in small GI tract pinch biopsies, due to the process of biopsy collection, which can result in the physical disruption of the epithelial barrier by the forceps during the biopsy procedure itself and due to the small size and orientation of the biopsies. We have demonstrated, however, that a local tissue response consisting of the infiltration of polymorphonuclear neutrophils (PMNs) in the LP of the GI tract is an excellent surrogate marker for epithelial damage and local microbial translocation $(6,29,30)$. Therefore, using immunohistochemistry and quantitative image analysis, we analyzed GI tract damage by measuring the extent of myeloperoxidase ${ }^{+}\left(\mathrm{MPO}^{+}\right)$PMNs within the LP (Figure 3). We observed a significant increase in the abundance of PMNs in FI/II patients compared with HIV- individuals (mean percentage area of PMNs in LP AHI, FI/II: $0.26 \%$ vs. HIV $: 0.032 \% ; P=0.017$ ) and in the FIII+ patients compared with HIV- $^{-}$individuals (mean percentage area of PMNs in LP AHI, FIII+: $0.16 \%$ vs. HIV-: $0.032 \% ; P=0.0076$ ) (Figure 3A). As expected, the number of PMNs in the LP of untreated chronically infected individuals was much higher than that found in the context of AHI (mean percentage area of PMNs in LP AHI, FI/II: $0.26 \%$ vs. CHI: $0.93 \% ; P=0.001$ and FIII+: $0.16 \%$ vs. CHI: $0.93 \% ; P=0.0004$ ).

We next investigated the extent of local immune activation within the LP of the GI tract by measuring the total cells that were Ki- $67^{+}$in the LP (Figure 4, A and B). During AHI, there was a significant increase in Ki-67 cells in the FIII+ group compared with HIV- individuals (mean percentage area of Ki- $67^{+}$cells in LP AHI, FIII+: $2.90 \%$ vs. HIV $: 1.05 \% ; P<0.0001$ ) and with chronically infected individuals (both cART treated and untreated) compared with $\mathrm{HIV}^{-}$individuals (mean percentage area of Ki-67+ cells in LP, CHI treated: $1.99 \%$, CHI untreated: $4.18 \%$ vs. HIV-: $1.05 \% ; P=0.049$ and $P<0.0001$, respectively) (Figure 4A). We observed a slight increase between AHI FI/II and HIV- individuals, but this difference was not statistically significant. Consistent with the early identification of FI/II individuals, the number of Ki- $67^{+}$cells in the FIII+ group was significantly higher than that observed in the FI/II group (mean percentage area of Ki- $67^{+}$cells in LP, AHI FIII+: 2.90 vs. AHI FI/II: $\left.2.18 ; P=0.0024\right)$.

We next determined the extent of local GI tract inflammation by quantifying (as the percentage area of the LP) the expression of the proinflammatory cytokine, TNF- $\alpha$ (Figure 4C); the type I IFN-responsive gene product, Mx1 (Figure 4D); IL-17 (Figure 4E); and indoleamine 2,3-dioxygenase-1 (IDO-1) (Figure $4 \mathrm{~F})$ during $\mathrm{AHI}$. The number of TNF- $\alpha^{+}$cells was significantly increased during AHI compared with levels seen in $\mathrm{HIV}^{-}$individuals (mean percentage area of TNF- $\alpha^{+}$, FI/II: $0.38 \%$, FIII+: $0.47 \%$ vs. HIV-: $0.1 \%$; $P=0.0006$ and $P=0.0005$, respectively). While the expression of Mx1 was heterogeneous within the 
A

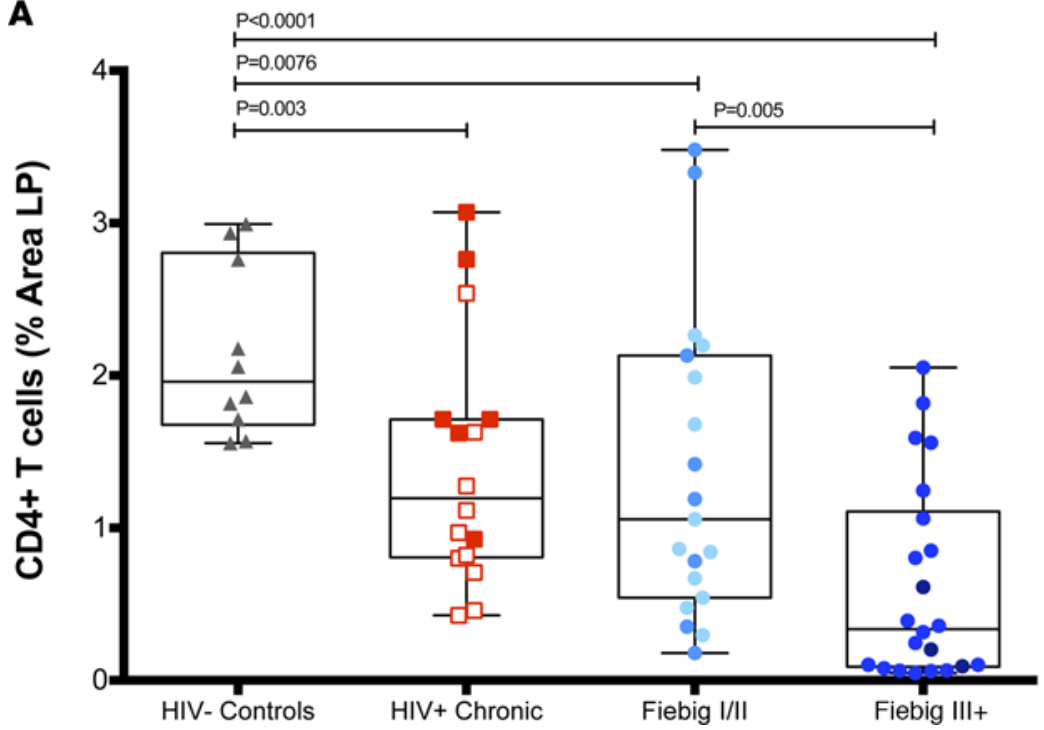

B

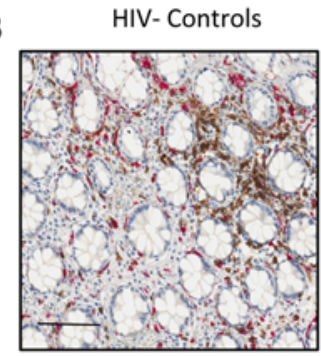

Fiebig I/II

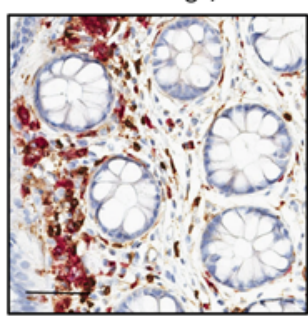

HIV+Chronic

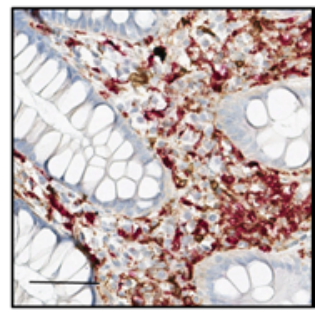

$>$ Fiebig III

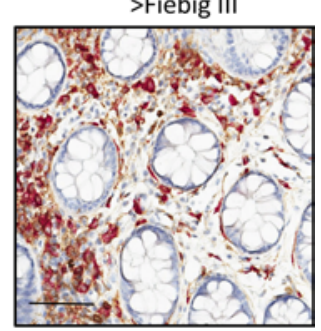

Figure 2. CD4 ${ }^{+} \mathbf{T}$ cells in the lamina propria of acute HIV-infected participants. Sigmoid biopsies were stained for CD4 (brown) and CD68/CD163 (red). The $C D 4^{+} T$ cell population is determined by subtraction of the myeloid population (red) from the total CD4+ cell population. The percentage area of the lamina propria (LP) that stained for CD4+ T cells was quantified in $\mathrm{HIV}^{-}(n=10)$, HIV chronic $(n=16)$, Fiebig I/II $(\mathrm{FI} / \mathrm{II})(n=19)$, and Fiebig III-V (FIII+) $(n=22)$ patients at the first visit (A). Fl/II patients presented varying levels of depletion, while the FIII+ participants manifested a drastic decrease of the $\mathrm{CD}^{+} \mathrm{T}$ cells in the LP at the first visit. Representative images for HIV- control, $\mathrm{HIV}^{+}$chronic, and the different Fiebig groups are shown in B. Each point represents the median of a minimum of 3 quantified biopsies per participant. Within the chronic patients, the open squares indicate untreated participants and the closed squares indicate treated participants. Within the $\mathrm{Fl} / \mathrm{Il}$ group, lighter blue dots represent Fl patients and darker blue dots represent the FII patients. Within FIII+ group, lighter blue dots represent FIII+ patients and darker dots represent FIV and FV patients. Scale bar: $100 \mu \mathrm{m}$. Following linear mixed-effects (Ime) analyses, post-hoc Ime contrasts and pairwise nonparametric (Wilcoxon) tests were performed. Benjamini-Hochberg adjusted $P$ values are reported. Data are presented as box-and-whisker plots with the lower and upper limits (bounds) of the box representing Q1 (25th percentile) and Q3 (75th percentile), respectively. The median (Q2, 50th percentile) is represented by the horizontal line within the box. Whiskers, delineated as horizontal stems, extend to the limits of the data. Data points (superimposed) are "jittered" to avoid overplotting.

different AHI groups, it was clearly elevated compared with levels found in $\mathrm{HIV}^{-}$individuals (mean percentage area of $\mathrm{Mx}^{+}$cells: FI/II: $6.67 \%$, FIII+: $2.52 \%$ vs. HIV ${ }^{-}: 0.08 \% ; P=0.002$ and $P=0.0004$, respectively). The expression of IL-17 in participants with AHI was unchanged compared with HIV- individuals (mean percentage area of IL- $17^{+}$cells in LP, AHI FI/II: $1.30 \%$, FIII+: $1.09 \%$ vs. HIV ${ }^{-}: 1.21 \% ; P=0.760$ and $P=0.670$, respectively) (Figure 4E). However, untreated participants with $\mathrm{CHI}$ had significantly higher expression of total IL-17 in the LP compared with FI/II and FIII+ groups (mean percentage area of IL-17 $7^{+}$ cells in LP, AHI FI/II: $1.30 \%$, AHI FIII+: $1.09 \%$ vs. CHI: $2.07 \% ; P<0.001, P<0.001$, respectively). Since inflammation and type I IFNs can also induce immunomodulatory enzymes such as the tryptophan-catabolizing enzyme, IDO-1, we quantified IDO-1 expression in the LP (Figure 4F) and observed an increase in $\mathrm{CHI}$ patients and in the $\mathrm{FI} / \mathrm{II}$ and $\mathrm{FIII}+$ groups compared with $\mathrm{HIV}^{-}$individuals (percentage area of IDO-1 cells in LP, FI/II: $1.99 \%$, FIII+: $1.25 \%$, CHI: $1.51 \%$ vs. HIV $: 0.46 \% ; P=0.029, P=0.002$, and $P=0.0009$, respectively). Collectively, these data highlight the dynamic and progressive nature of host immune responses and pathology in the GI tract during AHI.

Effect of early cART during AHI on GI tract damage, inflammation/immune activation, and CD4+ T cell reconstitution. After the initiation of CART, we observed a decrease of PMNs cells in the LP of individuals in the FI/II group; however, this was not statistically significantly, likely due to the low number of patients followed through 96 weeks of cART (mean percentage area PMNs in LP AHI FI/II, week 0: 0.26\% vs. week 24 cART: $0.05 \%$, week 96 cART: $0.089 \%, P=0.095$ and $P=0.303$, respectively) (Figure $5, \mathrm{~A}$ and C). We observed some variation among participants in the PMN number after treatment (Figure 5, B and D). Of the 15 patients who had biopsies that could be evaluated for PMN infiltration at the 3 visits (week 0 , 24, and 96), 8 had a significant decrease in their colonic PMN numbers (with slopes from -0.137 to -1.693 , $P=0.0006$ to $P=0.0148)$. The other 7 patients either had PMN numbers that were unchanged $(n=5)$ or increased $(n=2)$ during follow-up. Taken together, these results did not show a significant decrease in GI 
A

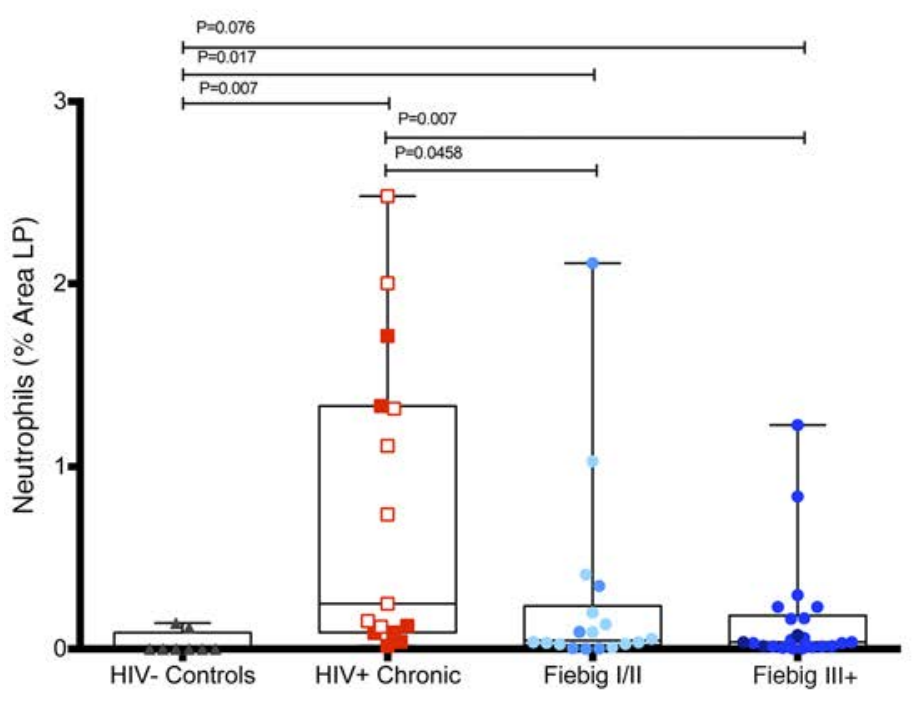

B

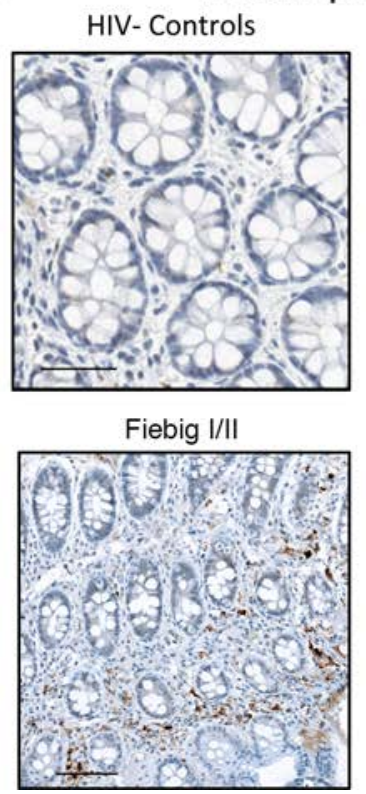

Neutrophils (MPO) HIV+ Chronic

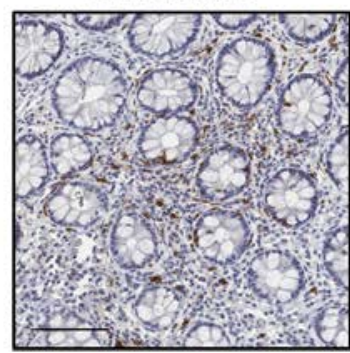

Fiebig III+

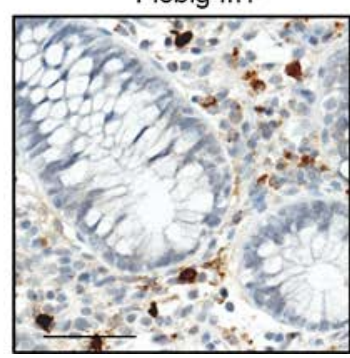

Figure 3. Increased gastrointestinal tract damage in early acute HIV-infected patients. Sigmoid biopsies were stained for myeloperoxidase (MPO), and the percentage area of neutrophils at the first visit was quantified. Each point represents the median percentage area of the lamina propria (LP) occupied by neutrophils quantified in a minimum of 3 biopsies for each participant. Notice the significant increase of neutrophils in Fiebig $1 / I I$ (FI/II) $(n=18)$ and Fiebig III-V (FIII+) ( $n=22)$ patients compared with HIV- controls $(n=10)(\mathbf{A})$. Representative images of MPO-stained biopsies of $\mathrm{HIV}^{-}$control, $\mathrm{HIV}^{+}$chronically infected, $\mathrm{FI} / \mathrm{II}$, and FIII+ participants are shown in B. Each point represents the median of a minimum of 3 quantified biopsies per participant. Within the chronic patient $(n=16)$, the open squares indicate untreated participants and the closed squares indicate treated participants. Within the FI/II group, lighter blue dots represent FI patients and darker blue dots represent the FII patients. Within FIII+ group, lighter blue dots represent FIII+ patients and darker dots represent FIV and FV patients. Scale bar: $100 \mu \mathrm{m}$. Following linear mixed-effects (Ime) analyses, post-hoc Ime contrasts and pairwise nonparametric (Wilcoxon) tests were performed. Benjamini-Hochberg adjusted p values are reported. Data are presented as box-and-whisker plots with the lower and upper limits (bounds) of the box representing Q1 (25th percentile) and Q3 (75th percentile), respectively. The median (Q2, 50th percentile) is represented by the horizontal line within the box. Whiskers, delineated as horizontal stems, extend to the limits of the data. Data points (superimposed) are "jittered" to avoid overplotting.

tract damage, as measured by PMN infiltration, in either AHI Fiebig groups after cART treatment $(P=0.375)$. While the number of PMNs did not significantly decrease over the 96 weeks of follow-up in the FI/II group, the number of PMNs at week 96 was not significantly different compared with the HIVindividuals (mean percentage area PMNs in LP, $\mathrm{HIV}^{-}: 0.03 \%$ vs. AHI FI/II week 96: 0.089\%, $P=0.081$ ); however, there was still a significant difference when comparing the FIII+ group at week 96 with the HIVindividuals (mean percentage area of PMNs in LP HIV-: 0.03 vs AHI FIII+: 0.30, $P=0.027$ ). The elevated mean percentage area of PMNs in the FIII+ group at week 96 was driven by one patient who had much higher numbers of PMNs (percentage area PMNs in LP: $1.71 \%$ ) compared with the remaining FIII+ individuals (mean percentage area of PMNs in LP: $0.10 \%$ with patient removed, mean percentage area PMNs in LP HIV $: 0.03 \%$ vs. AHI FIII+: $0.10 \%, P=0.027$ ). Importantly, the patient with the elevated PMN levels at week $96 \mathrm{cART}$ also had a positive viral load at this time point, suggesting treatment failure and recurrent GI tract damage. Collectively, these data suggest that early cART may lead to an improvement in GI tract integrity in patients who adhere to their medications; however, damage may persist even after 96 weeks of treatment in some patients.

Next, we sought to ascertain the effect of cART on levels of immune activation and inflammation within the LP of the large intestine (Figure 6). After 24 weeks of treatment, the number of Ki- $67^{+}$cells significantly decreased in both Fiebig groups $(P=0.016)$, reaching baseline levels after 96 weeks of cART compared with uninfected individuals (mean percentage area of $\mathrm{Ki}-67^{+}$cells in LP AHI, week 96 cART FI/ II: $1.05 \%$, week 96 cART FIII+: $0.98 \%$ vs. HIV $: 1.05 \% ; P=0.887$ and $P=0.668$, respectively) (Figure 6 , $A$ and $B)$. To determine the effect of cART on levels of inflammation within the LP, we first quantified the number of cells that were expressing the proinflammatory cytokine, TNF- $\alpha$. After the initiation of cART, the number of TNF- $\alpha^{+}$cells decreased in the FI/II group (mean percentage area of TNF- $\alpha^{+}$cells FI/II at week 0: $0.38 \%$ vs. week $24: 0.08 \%$ and week $96 \%: 0.17 ; P=0.0065$ and $P=0.020$, respectively) (Figure $6 \mathrm{C}$ ). 
A

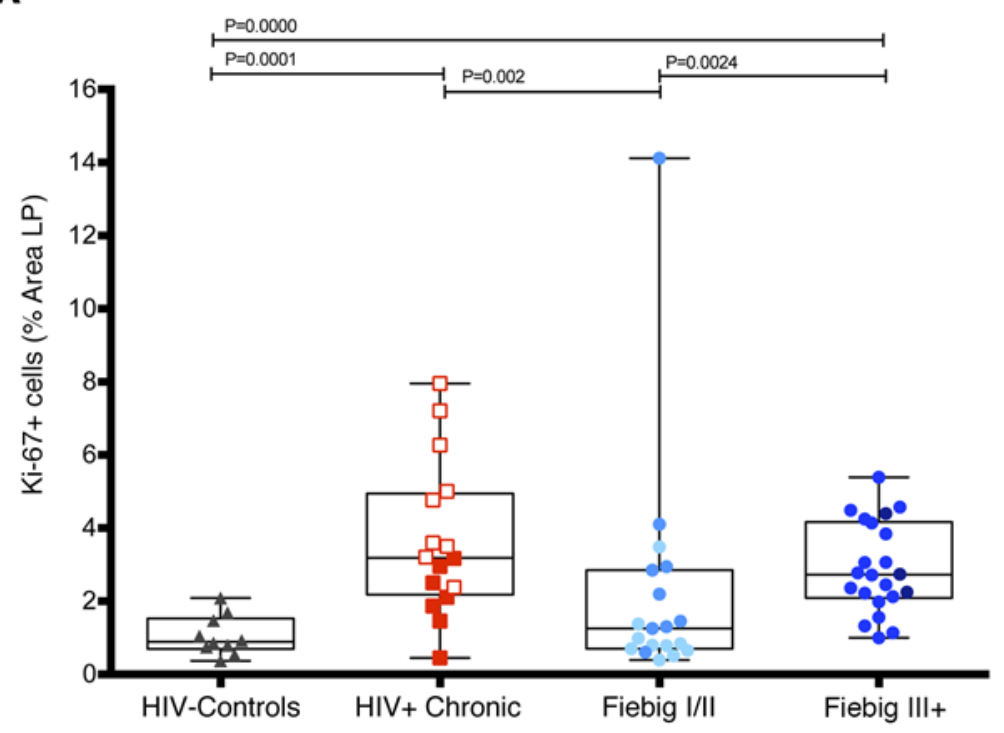

B
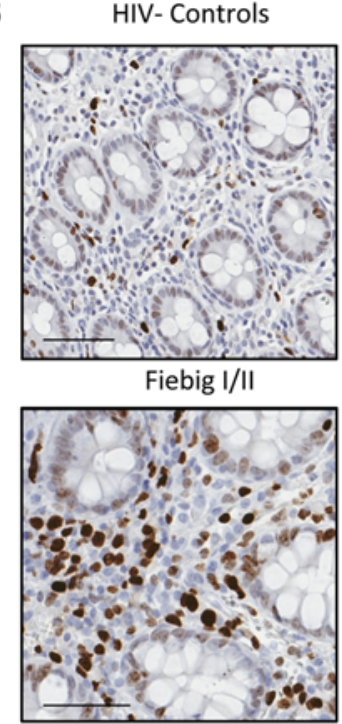

HIV+ Chronic

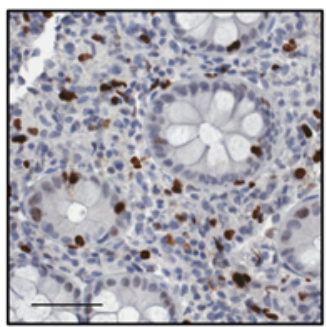

Fiebig III+

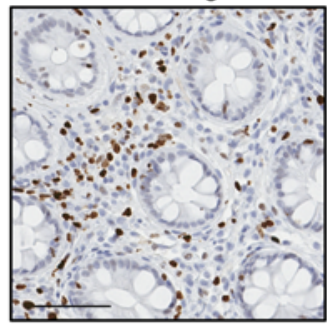

C

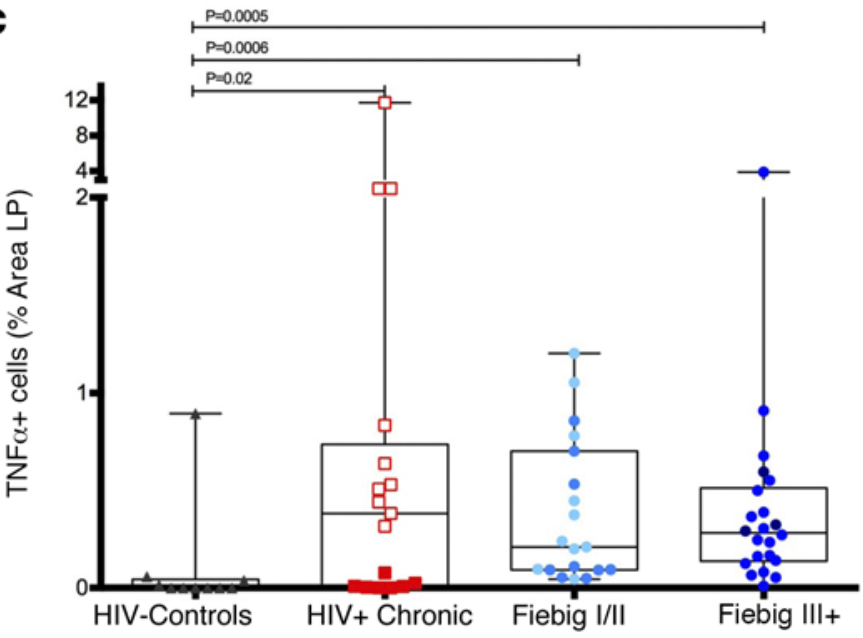

E

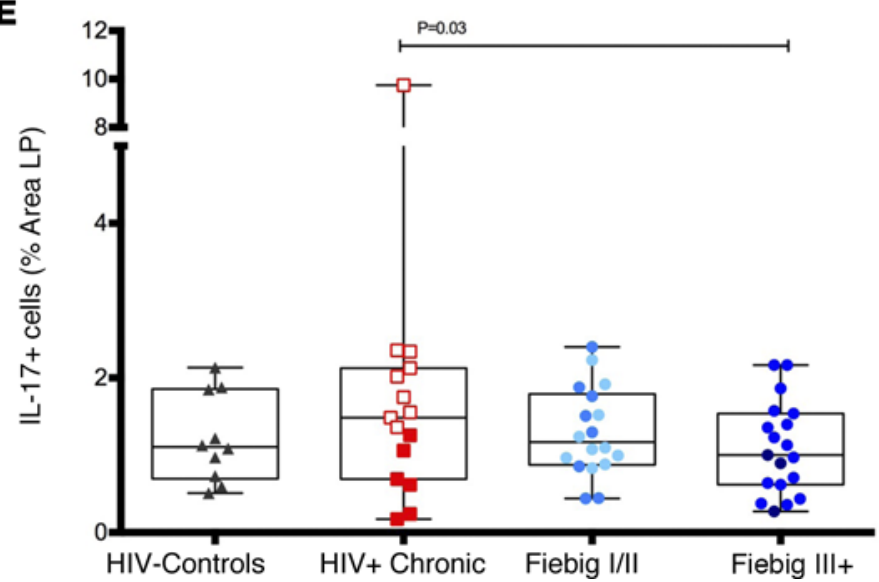

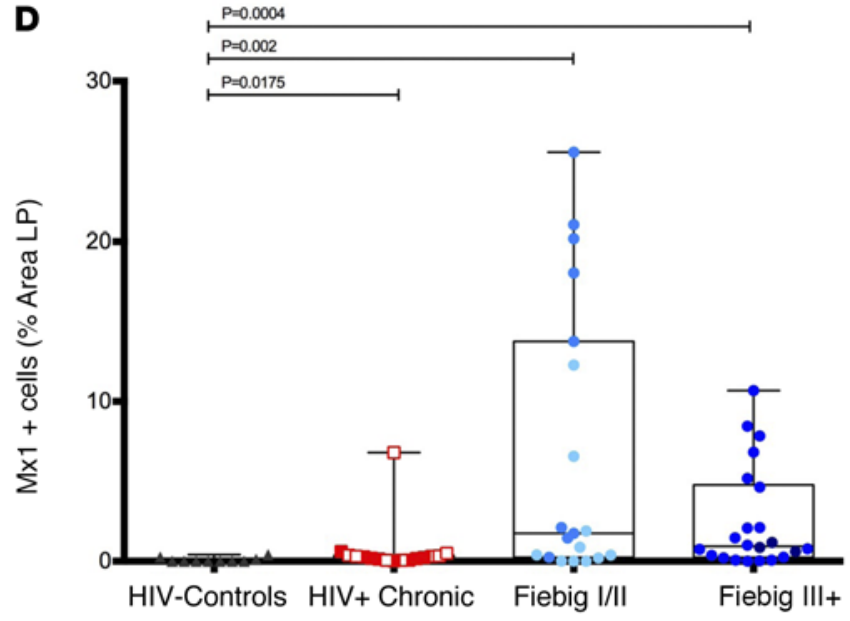

$\mathbf{F}$

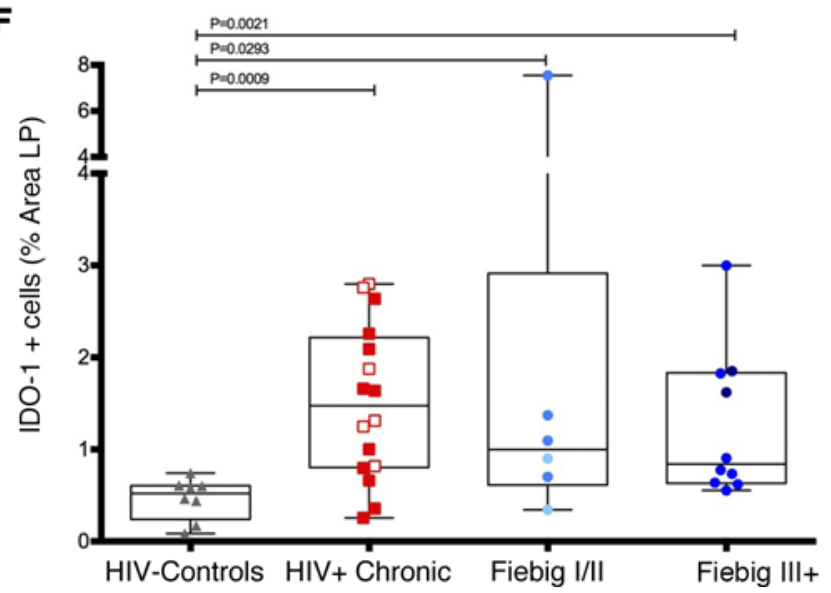


Figure 4. Inflammation and activation markers in acute HIV-infected participants. Sigmoid biopsies were stained with Ki-67 to quantify the number cells in the lamina propria (LP) with evidence of recent cell division (A). Representative images of Ki-67-stained biopsies of HIV- $(n=10)$, HIV+ chronic $(n=$ 16), Fiebig I/II (FI/II) $(n=19)$, and Fiebig III-V (FIII+) $(n=22)$ patients (B). TNF+ (C), MX dynamin like GTPase $1^{+}(\mathrm{Mx} 1)(\mathbf{D}), \mathrm{IL}-17^{+}(\mathrm{FI} / \mathrm{II} n=18, \mathrm{FIII}+n=19)(\mathbf{E})$, and indoleamine 2,3-dioxygenase ${ }^{+}(\mathrm{IDO}-1)(\mathbf{F})$ cells $(\mathrm{FI} / \mathrm{II} n=6$, FIII $n=10$ ) were quantified in sigmoid biopsies in acute HIV-infected patients. Each point represents the median of a minimum of 3 quantified biopsies per participant. Within the chronic patient, the open squares indicate untreated participants and the closed squares indicate treated participants. Within the FI/II group, lighter blue dots represent FI patients and darker blue dots represent the FII patients. Within FIII+ group, lighter blue dots represent FIII+ patients and darker dots represent FIV and FV patients. Scale bar: $100 \mu \mathrm{m}$. Following linear mixed-effects (Ime) analyses, post-hoc Ime contrasts and pairwise nonparametric (Wilcoxon) tests were performed. Benjamini-Hochberg adjusted $P$ values are reported. Data are presented as box-and-whisker plots with the lower and upper limits (bounds) of the box representing Q1 (25th percentile) and Q3 (75th percentile), respectively. The median (Q2, 50th percentile) is represented by the horizontal line within the box. Whiskers, delineated as horizontal stems, extend to the limits of the data. Data points (superimposed) are "jittered" to avoid overplotting.

The FIII+ group, by contrast, did not show significant changes in TNF- $\alpha$ expression until 96 weeks of treatment $(P=0.020)$. After 96 weeks of cART, the number of TNF- $\alpha^{+}$cells went back to levels similar to those of $\mathrm{HIV}^{-}$individuals for both AHI groups (mean percentage area of TNF- $\alpha^{+}$cells at week $96 \mathrm{cART}$, FI/II: $0.17 \%$, FIII+: $0.10 \%$ vs. HIV $: 0.10 \% ; P=0.089$ and $P=0.058$, respectively) (Figure $6, \mathrm{C}$ and D).

To further assess the effect of cART on levels of inflammation within the LP, we quantified the number of cells that were expressing the type I IFN-responsive gene product, Mx1. The number of $\mathrm{Mx} 1^{+}$cells decreased significantly after the initiation of cART in both Fiebig groups (mean percentage area of $\mathrm{Mx}^{+}$ cells at week 0 cART, FI/II: $6.67 \%$, FIII+: $2.52 \%$ vs. FI/II week 24: $0.07 \%$ and FIII+ week $24: 0.13 \%$; $P=0.0073$ and $P=0.0074$, respectively) (Figure $6, \mathrm{E}$ and $\mathrm{F}$ ). The resolution of type I IFN inflammation appeared quite rapid, as after 24 weeks of treatment the number of $\mathrm{Mx}^{+}$cells had returned to levels comparable to those of the $\mathrm{HIV}^{-}$individuals for both Fiebig groups (mean percentage area of $\mathrm{Mx} 1^{+}$cells, FI/ II week 24: $0.07 \%$ and FIII+ week $24: 0.13 \%$ vs. HIV : $0.08 \% ; P=0.078$ and $P=0.18$, respectively). The expression of IL-17 in the LP, on the other hand, did not change from week 0 of cART for either the FI/II (mean percentage area of IL-17 $7^{+}$cells in LP, week 0 FI/II: $1.10 \%$, week 24 cART: $1.10 \%$, week 96 cART: $1.0 \% ; P=0.485$ and $P=0.386$, respectively) or FIII+ groups (week $0 \mathrm{FIII+:} 1.07 \%$, week $24 \mathrm{cART}: 1.6 \%$, and week 96 cART: $1.3 \% ; P=0.264$ and $P=0.690$, respectively) (Figure 6, G and $\mathrm{H}$ ).

Finally, we assessed the effect of early cART on $\mathrm{CD}^{+} \mathrm{T}$ cell levels in the LP in our AHI cohort. After 24 weeks of cART, the number of $\mathrm{CD}^{+} \mathrm{T}$ cells in the LP was significantly decreased in FI/II individuals compared with week 0 (mean percentage area of $\mathrm{CD}^{+} \mathrm{T}$ cells in LP FI/II, week 0: $1.35 \%$ vs. week 24 cART: $0.56 \% ; P=0.0078$ ) (Figure 7A). However, the FIII+ individuals showed no significant difference after 24 weeks of cART compared with week 0 (mean percentage area of $\mathrm{CD} 4^{+} \mathrm{T}$ cells in LP, week 0: 0.62\% vs. week 24 cART: $0.52 \% ; P=0.672$ ) (Figure 7B). Importantly, while after 96 weeks of cART, FI/II individuals showed a modest but significant increase in the number $\mathrm{CD}^{+} \mathrm{T}$ cells in the LP compared with the 24 week cART time point (mean percentage area of $\mathrm{CD}^{+} \mathrm{T}$ cells in LP week 24 cART: $0.56 \%$ vs. 96 week cART: 0.89\%; $P=0.0306$ ), the number of $\mathrm{CD} 4^{+} \mathrm{T}$ cells at 96 weeks of cART was, however, still marginally significantly lower than that found in $\mathrm{HIV}^{-}$individuals (mean percentage area of $\mathrm{CD}^{+} \mathrm{T}$ cells in LP FI/II week 96 cART: $1.36 \%$ vs. HIV $: 2.14 \% ; P=0.043$ ) (Figure 7A). By contrast, the number of CD4 ${ }^{+} \mathrm{T}$ cells in the LP of the FIII+ group between 24 and 96 weeks of cART (mean percentage area of CD4 $4^{+} \mathrm{T}$ cells in LP, week 24 cART: $0.51 \%$ vs. week 96 cART: $0.89 \% ; P=0.31$ ) and after 96 weeks of cART (mean percentage area of CD4 ${ }^{+} \mathrm{T}$ cells in LP FIII+ week 96 cART: $0.89 \%$ vs. HIV $: 2.14 \% ; P=0.0001$ ) continued to be significantly lower compared with that found in HIV- individuals (Figure 7B). Furthermore, at 96 weeks of cART, the FI/II individuals showed a significant difference in the number of $\mathrm{CD}^{+} \mathrm{T}$ cells in the LP compared with the FIII+ group (mean percentage area of $\mathrm{CD}^{+}$T cells in LP, week 96 cART FI/II: 1.36 vs. week 96 cART FIII+: $\left.0.89 ; P=0.04\right)$, suggesting an incremental benefit for very early cART treatment. Finally, after initiation of the treatment, the abundance of myeloid lineage cells within the LP did not change in any group (data not shown).

\section{Discussion}

GI tract pathologies, including early depletion of $\mathrm{CD}^{+} \mathrm{T}$ cells in the LP and damage to the GI tract epithelial barrier, are known to be characteristic of HIV-1 infection (13). We initially hypothesized that such effects might be mitigated by the early initiation of cART, reasoning that control of viral replication at the earliest time point clinically feasible would have a positive effect on the loss of CD4 ${ }^{+} \mathrm{T}$ cells in the effector sites of GI tract tissues. We accordingly investigated the effect of early initiation of cART in acute HIV-1-infected patients in Thailand enrolled in the RV254 study. 


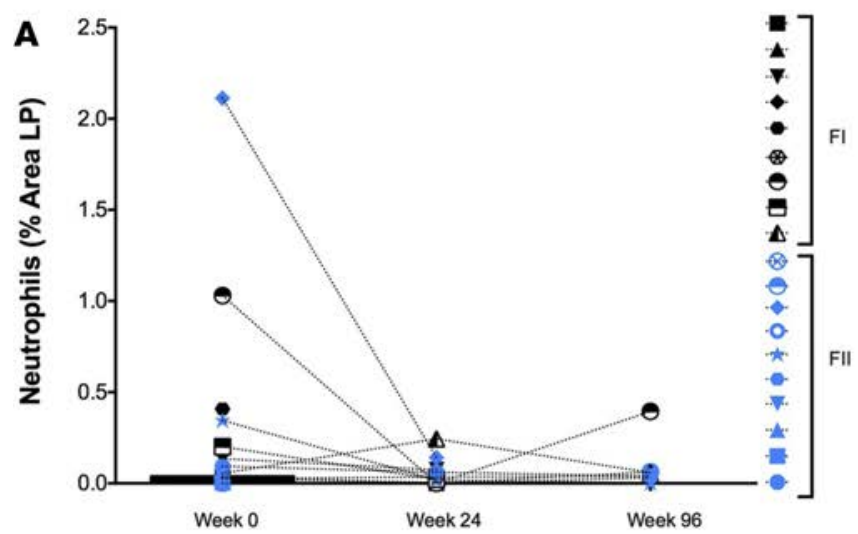

C

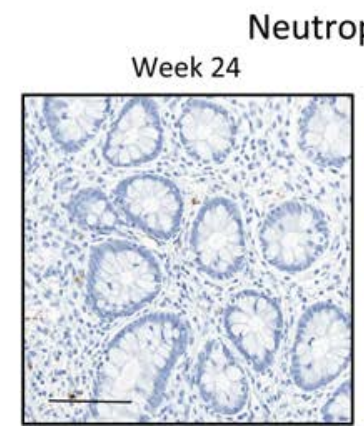

phils (MPO)

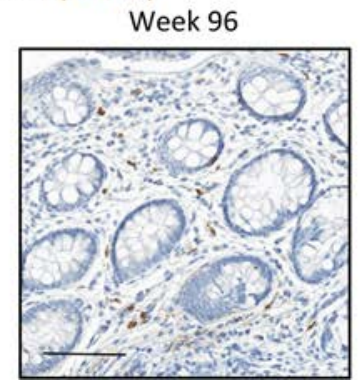

B

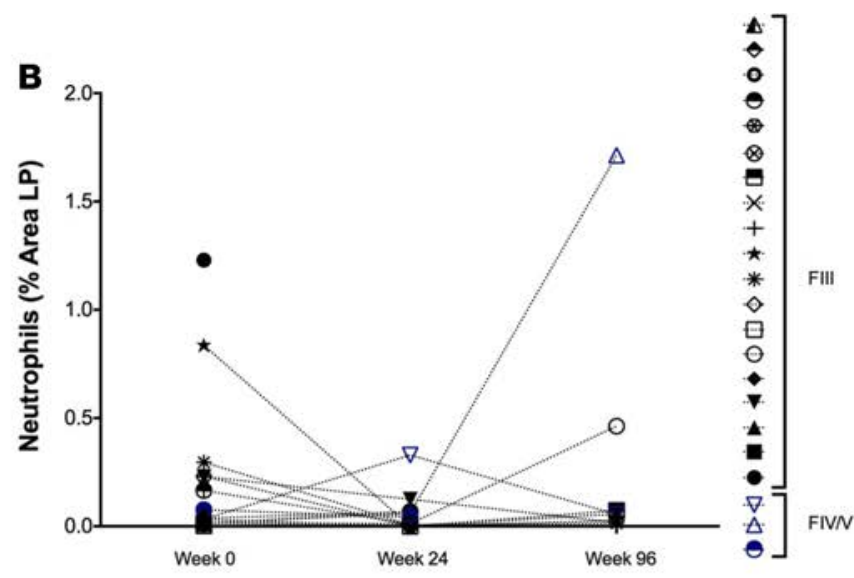

D

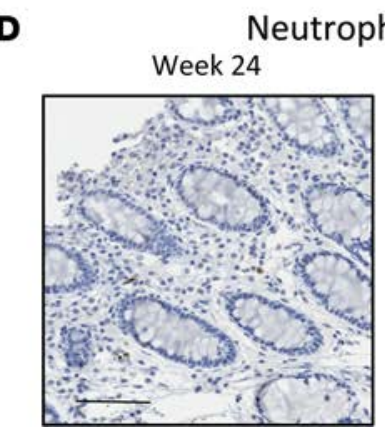

Week 96

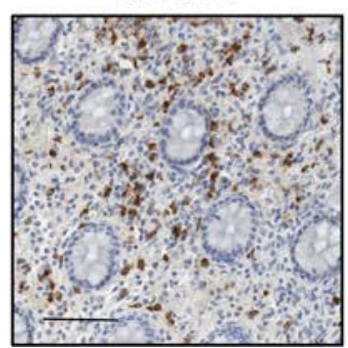

Figure 5. Longitudinal quantification of damage to the gastrointestinal tract after $\mathbf{2 4}$ and $\mathbf{9 6}$ weeks of antiretroviral treatment. Sigmoid biopsies were stained for myeloperoxidase (MPO) as a surrogate marker of the mucosal damage in Fiebig I (FI) and II (FII) participants (week $0, n=18$ ) (A) and in Fiebig III (FIII) and IV/V (FIV/V) patients (week $0, n=22)$ (B) 24 (FI/II and FIII+, $n=13$ ) and 96 weeks (FI/II, $n=7 ; \mathrm{FIII+,} n=13$ ) after the initiation of combination antiretroviral therapy (cART). Each symbol represents a patient. FII is distinguishable from FI by the light-blue colored symbols, and FIV/V is distinguishable from FIII by the dark-blue colored symbols. Representative images of Fl participants at 24 weeks and 96 weeks after treatment are shown in C. One Fiebig $V$ patient showed an increase of neutrophils at 96 weeks (D); this patient had a detectable blood viral load at this same time point. Each point represents the median of a minimum of 3 quantified biopsies per participant. Scale bar: $100 \mu \mathrm{m}$. Following repeated-measures and linear mixed-effects (Ime) analyses, post-hoc Ime contrasts and pairwise nonparametric (Wilcoxon) tests were performed. Benjamini-Hochberg adjusted $P$ values are reported.

Here, we demonstrate that individuals identified during early AHI already had extensive evidence of GI tract pathologies, including GI tract damage, associated inflammation, and immune activation, and the loss of CD4 ${ }^{+} \mathrm{T}$ cells from the LP effector site of the GI tract. These data are consistent with previous observations made in SIV-infected nonhuman primates (31-33) and suggest (a) that the pathologic effects of HIV-1 infection in the GI tract begin remarkably early during acute infection and (b) that these initial effects are not preventable, even when cART is commenced at the very earliest feasible time point clinically. Notwithstanding these sobering facts, it is highly encouraging that cART does result in reversal of many other key features of early GI tract pathology, including resolution of GI tract inflammation and immune activation, and GI tract epithelial damage with microbial translocation in some patients (as measured by neutrophil infiltration). Notably, these are features that persist in many HIV-infected individuals who are treated later in the disease course $(6,7,34,35)$. The importance of restoring GI tract integrity and attenuating GI tract inflammation in patients treated in early acute infection may help explain why patients identified and treated at the earliest Fiebig stages have reduced HIV reservoirs $(36,37)$, particularly since residual inflammation during cART is thought to be a critical driver of HIV persistence (38). Thus, reducing inflammation may be an effective way to obstruct the maintenance of HIV reservoirs in cART-suppressed individuals.

It is important to note that we identified several individuals during the early FI/II stage who did not have evidence for $\mathrm{CD}^{+} \mathrm{T}$ cell depletion in the LP prior to cART but who did subsequently show CD4 ${ }^{+}$ $\mathrm{T}$ cell depletion after 24 weeks of cART. These data suggest that cART intervention simply cannot be feasibly administered quickly enough to prevent the early consequences of HIV-1-mediated destruction of $\mathrm{CD}^{+} \mathrm{T}$ cells within the LP effector compartment of the gut. Indeed, while our previous analysis of many of these same individuals suggested significant $\mathrm{CD}^{+} \mathrm{T}$ cell recovery in the GI tract, as assessed by flow 
A
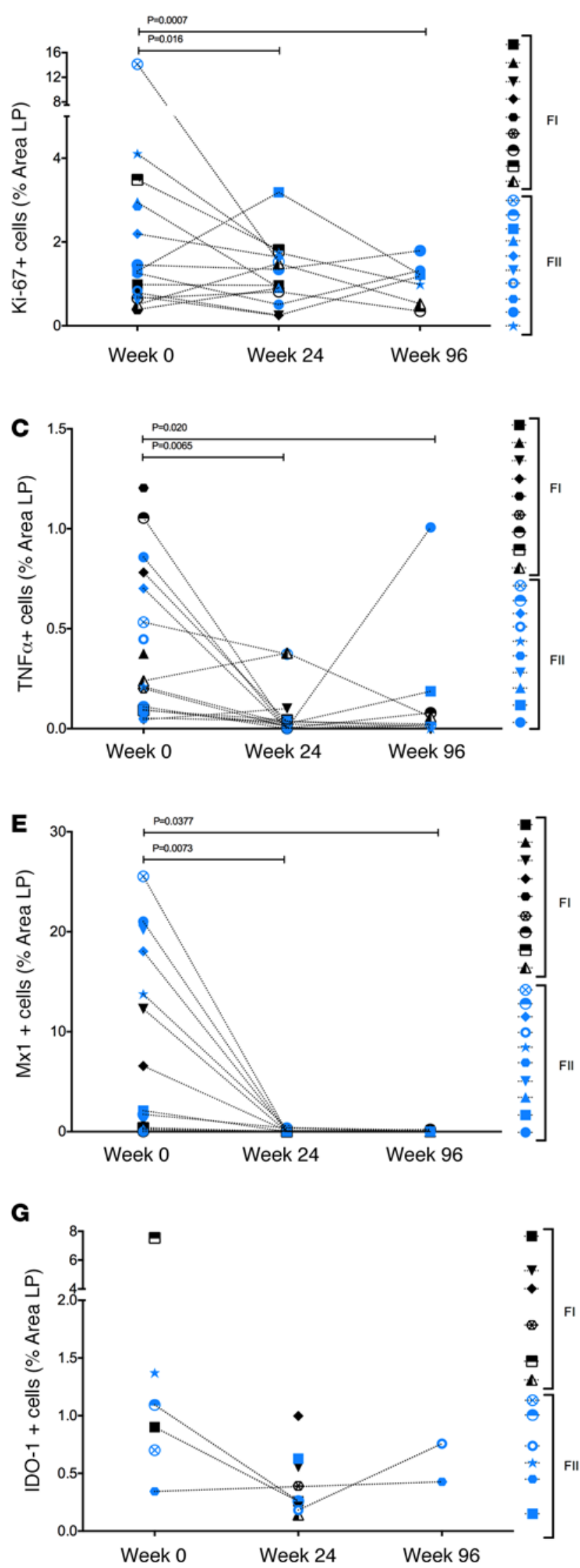
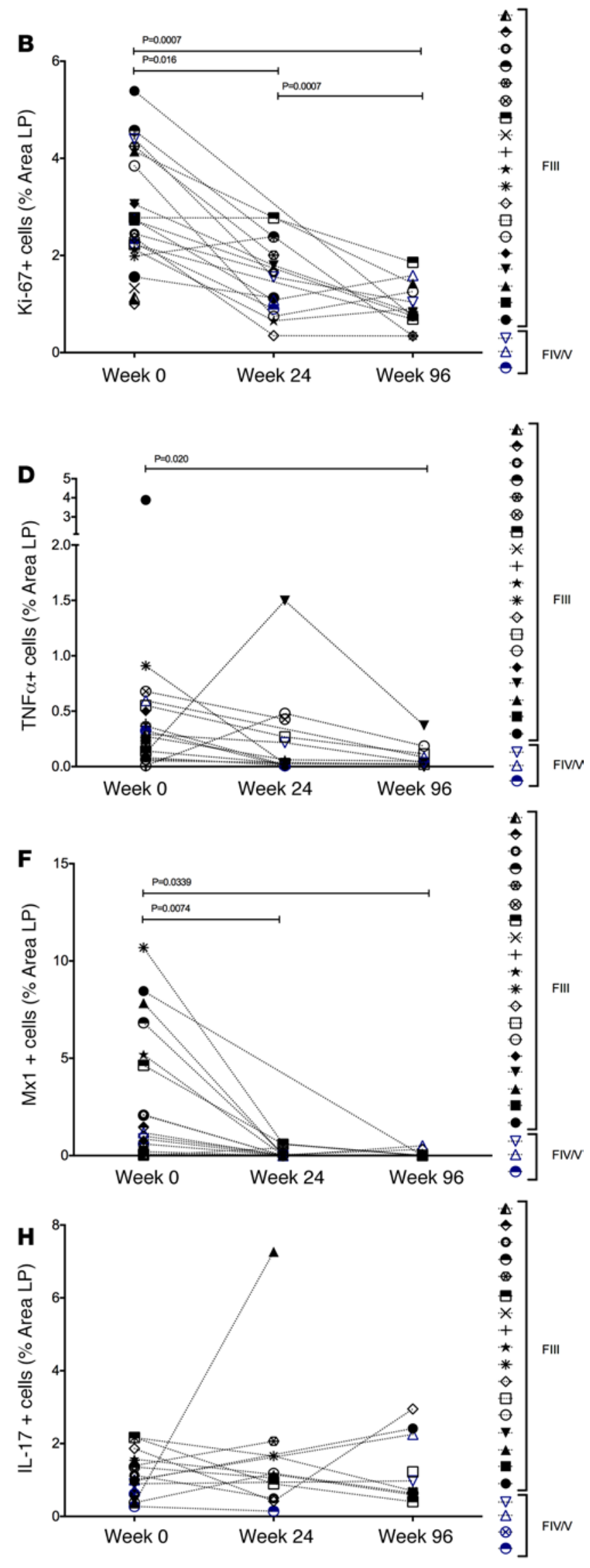
Figure 6. Inflammation and immune activation within the gastrointestinal tract of acute HIV-infected and treated participants. Ki-67 ${ }^{+}$cells were quantified in the lamina propria (LP) after 24 and 96 weeks of treatment in the Fiebig I/II (FI/II) group (week $0, n=19$; week 24, $n=14$; week 96, $n=7$ ) (A) and in the Fiebig III (FIII), IV/V group (week $0, n=22$; week 24, $n=13$; week 96, $n=12$ ) (B). TNF+ cells were quantified in the LP after 24 and 96 weeks of treatment in the FI/II group (week 0, $n=19$; week 24, $n=14$; week 96, $n=8$ ) (C) and in the FIII, IV/V group (week 0, $n=22$; week 24, $n=13$; week 96, $n=9$ ) (D). Sigmoid biopsies of FI/ II (week 0, $n$ = 19; week 24, $n=14$; week 96, $n=6$ ) (E) and FIII+ (week $0, n=22$; week 24, $n=13$; week 96, $n=8$ ) (F) participants were stained and quantified for MX dynamin like GTPase 1 (Mx1) before and 24 weeks and 96 weeks after initiation of combination antiretroviral therapy (cART). Indoleamine 2,3-dioxygenase ${ }^{+}$ (IDO-1) (G) and IL-17+ $(\mathbf{H})$ cells were quantified in the LP of sigmoid biopsies in FI/II (week $0, n=18$; week 24, $n=12$; week 96, $n=5$ ) and FIII+ participants (week $0, n=19$; week 24, $n=10$; week 96, $n=10$ ) at the first visit (week 0 ) and followed over time at 24 weeks and 96 weeks after treatment. Each symbol represents a patient. FII is distinguishable from FI by the light-blue colored symbols, and FIV/V is distinguishable from FIII by the dark-blue colored symbols. Each point represents the median of a minimum of 3 quantified biopsies per participant. Following repeated-measures and linear mixed-effects (Ime) analyses, post-hoc Ime contrasts and pairwise nonparametric (Wilcoxon) tests were performed. Benjamini-Hochberg adjusted $P$ values are reported.

cytometry (25), we demonstrate here by histological evaluation that CD4 ${ }^{+} \mathrm{T}$ cell levels in the LP effector site of the GI tract did not return to normal in most individuals treated during early AHI. This dichotomy suggests that the previously observed increases occurred within lymphoid tissue structures (e.g., lymphoid aggregates) and not within the effector sites of the gut. This is in concordance with several previous studies demonstrating that $\mathrm{CD} 4^{+} \mathrm{T}$ cells in the GI tract remained significantly lower than in $\mathrm{HIV}^{-}$controls, despite effective prolonged cART $(14,39-43)$. It is encouraging to note that several individuals treated during FI/ II approached $\mathrm{HIV}^{-}$baseline $\mathrm{CD} 4^{+} \mathrm{T}$ cell levels in the LP and demonstrated significant increases in $\mathrm{CD} 4^{+}$ $\mathrm{T}$ cells from the 24 to 96 week time point. Although additional follow up will be required to definitively determine the extent to which such recovery occurs in this cohort, this observation suggests that $\mathrm{CD} 4^{+} \mathrm{T}$ cell reconstitution may be possible at least in some individuals treated early.

Numerous studies have reported the importance of IL-17A in neutrophil recruitment, proliferation of epithelial cells, and production of epithelial tight junction proteins and antimicrobial defensins (44, 45). IL-17A is produced by numerous cells, including Th17 $\mathrm{CD} 4^{+} \mathrm{T}$ cells, and has been shown to be drastically decreased in the gut of HIV-1 chronically infected patients (46). The loss of multiple lineages of IL-17-producing lymphocytes, including $\mathrm{Th} 17 \mathrm{CD} 4^{+} \mathrm{T}$ cells that are critical for the maintenance of gut epithelial barrier integrity, has been associated with damaged mucosal barrier and microbial translocation as well as dysfunctional local innate and adaptive immune functions and disease progression (47-50). In this study, we were unable to measure IL-17A specifically, given that the polyclonal antibody used has been demonstrated to have some cross-reactivity to other IL-17 isoforms (notably IL-17F and to a lesser extent IL-17C, D and E). Nonetheless, we did not observe a decrease of total IL-17 expression in the LP of the GI tract during AHI in either Fiebig group, a finding consistent with those in our previous study utilizing flow cytometry to assess $\mathrm{T}$ cell population changes (25) and in a more recent study (51). The lack of loss of IL- $17^{+}$cells in FIII+ patients in our current study compared with the initial loss of Th17 cells reported in our previous study (25) could also be explained by the fact that IL-17A is produced by numerous cell types other than Th17 cells, e.g., CD8 ${ }^{+}$T cells, T cells, NK cells, NKT cells, LTi, neutrophils, and Paneth cells (52). Alternatively, early treatment with cART may result in the preservation of $\mathrm{CD}_{103^{+}} \mathrm{DC}$ s within the $\mathrm{LP}$ of the large intestine, as loss of such cells has been associated with the loss of IL-17- and IL-22-producing lymphocytes (50); however, due to limited biopsy size and sampling, we were unable to investigate this possibility. These results suggest that compensatory and/or redundant mechanisms exist for IL-17 expression and may be important for the resolution that we observed in the mucosal barrier of the gut.

The mechanisms for the lack of $\mathrm{CD} 4^{+} \mathrm{T}$ cell recovery in the $\mathrm{LP}$ are not fully understood but may be due in part to collagen deposition in the gut-inductive sites (53) and/or to the loss of expression of gut-homing receptors on circulating $\mathrm{CD} 4^{+} \mathrm{T}$ cells $(42,54)$. While additional studies will be required to completely understand the mechanisms and the clinical implications of continued $\mathrm{CD} 4^{+} \mathrm{T}$ cell depletion in the setting of cART, we did observe significantly decreased levels of local inflammation and immune activation in the GI tract with early cART treatment. Several previous studies have reported a decrease in peripheral $\mathrm{T}$ cell activation $(55,56)$ and a variety of plasma biomarkers of inflammation $(36,57,58)$ in individuals treated during acute infection; by inference, such changes might be associated with a reduced rate of disease progression and a decreased risk of non-AIDS comorbidities compared with those who initiate cART at a later time after infection $(13,59-62)$. If so, attenuation of GI tract pathologies could have a positive effect on the overall health of HIV-infected individuals treated early during AHI, as these individuals would be predicted to have fewer non-AIDS comorbidity events, with improved clinical outcomes linked to a better quality of life and life expectancy compared with individuals starting treatment later $(63,64)$. 
A

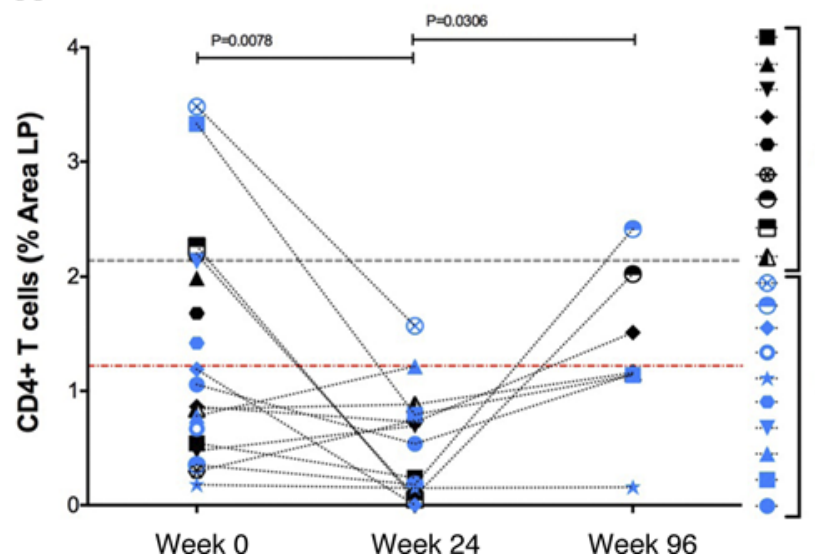

B

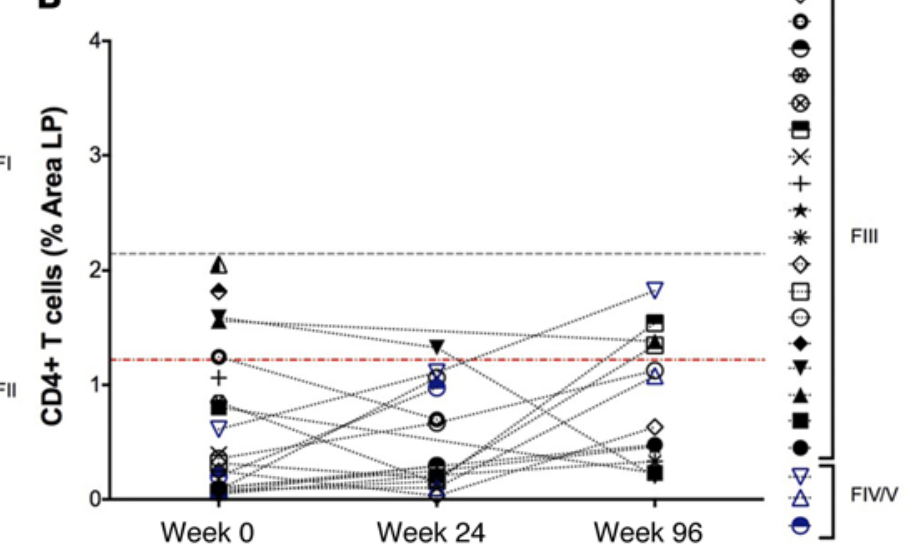

Figure 7. Effect of combined antiretroviral therapy on the CD4+ $\mathrm{T}$ cell population in the lamina propria of acute HIV-infected participants. Sigmoid biopsies were stained for CD4 and CD68/CD163. The CD4+ T cell population was determined by subtraction of the myeloid population from the total CD4 cell population. The percentage area of the lamina propria that stained for CD4+ $\mathrm{T}$ cells was quantified in Fiebig I/II (FI/II) participants (week $0, n=19$; week 24, $n=14$; week 96, $n=7$ ) (A) and in Fiebig III-V (FIII+) patients (week 0, $n=22$; week 24, $n=14$; week 96, $n=12$ ) (B) at the first visit (week 0) and again at 24 weeks and 96 weeks after treatment. The dashed lines represent the median of CD4+ T cells in healthy control participants (gray, $n=10$ ) and in chronic HIV-infected patients (red, $n=17$ ). Each symbol represents a patient. FII is distinguishable from FI by the light-blue colored symbols, and FIV/V is distinguishable from FIII by the dark-blue colored symbols. Each point represents the median of a minimum of 3 quantified biopsies per participant. Following repeated-measures and linear mixed-effects (Ime) analyses, post-hoc Ime contrasts and pairwise nonparametric (Wilcoxon) tests were performed. Benjamini-Hochberg adjusted $P$ values are reported.

\section{Methods}

Study participants. The RV254/SEARCH 010 study is an ongoing prospective, open-label study based in Bangkok, Thailand (clinicaltrials.gov identification NCT00796146). Blood samples from participants from clients from the Thai Red Cross Anonymous Clinic and the Silom Community Clinic were screened in real time by pooled NAT and sequential EIA, according to published methods (65). Participants who had positive NAT and nonreactive HIV IgG were enrolled in the RV254 cohort. Gut biopsies were performed by sigmoidoscopy on a volunteer basis (including 41 participants at baseline [before ART], 27 at 24 weeks after ART, and 18 at week 96 after ART). cART was initiated as part of enrollment in an accompanying local protocol (clinicaltrials.gov identification NCT00796263) approved by the Chulalongkorn University IRB. Treatment was started as soon as possible after enrollment (range 0-5 days). Subsequently, participants were randomized to 5-drug (MegaHAART, including tenofovir [TDF], emtricitabine [FTC] or lamivudine [3TC], efavirenz [EFV], raltegravir [RAL], and maraviroc [MVC]) ART vs. 3-drug (HAART with TDF, FTC/3TC, and EFV) ART. The 5-drug regimen was given as followed for the first 24 weeks: TDF, $300 \mathrm{mg}$ once daily; FTC, $200 \mathrm{mg}$ once daily, or 3TC, $300 \mathrm{mg}$ once daily; EFV, $600 \mathrm{mg}$ once daily; RAL, $400 \mathrm{mg}$ twice daily; and MVC, 600 mg twice daily; this was followed by simplification to 3 drugs, i.e., TDF, FTC/3TC, and EFV. In case of intolerance or resistance, EFV was discontinued, and the dose of MVC was adjusted to $300 \mathrm{mg}$ twice daily. Patients were initially screened for potential coinfections and at the time of each biopsy. In the FI/II group, at enrollment, 1 participant had gonorrhea proctitis, 4 had syphilis, and 2 were diagnosed with hepatitis B virus (HBV) infection. In the FIII+ group, 6 participants had syphilis and 1 had genital herpes at enrollment; another 2 participants had gonorrhea infection at 16 weeks and 24 weeks after enrollment. It is important to note that all participants with HBV infection received TDF and 3TC or FTC per the standard of care in Thailand. All study participants signed informed consent prior to study enrollment.

Diagnosis of AHI. Diagnosis of AHI was performed as described previously $(25,36)$. In brief, Thai AHI participants were enrolled if they fulfilled laboratory criteria for Fiebig stages I-V (28) as follows: FI: positive HIV RNA, negative p24 antigen, negative third generation IA; FII: positive HIV RNA, positive p24 antigen, negative third generation IA; FIII: positive third generation IA, negative Western blot; FIV: positive third generation EIA, indeterminate Western blot; and FV: positive third generation IA, positive Western blot except p31.

$v R N A$ quantification in plasma and tissue. HIV RNA in plasma was measured using the Roche Amplicor v 1.5 ultrasensitive assay with a lower quantification limit of 50 copies/ml (Roche Diagnostics). For gut 
tissue, 1-2 biopsy pieces frozen in RNAlater (Ambion) were weighed and then homogenized in AVL buffer (QIAamp Viral mini kit, catalog No. 52,904, QIAGEN), using a mini-mortar and pestle. Extraction was completed following kit instructions. The Siemens Quantiplex HIV-1 3.0 assay was used to measure HIV-1 RNA copy number. Results are expressed as copies/mg of tissue.

Immunohistochemistry. Immunohistochemical staining and quantitative image analysis were performed as previously described (66). In brief, immunohistochemistry was performed using a biotin-free polymer approach (Golden Bridge International) on 5 - $\mu \mathrm{m}$ tissue sections mounted on glass slides, which were dewaxed and rehydrated with double-distilled water. Multistaining of CD4/CD68/CD163 to quantify $\mathrm{CD}^{+} \mathrm{T}$ cells was performed as described previously (67). This multistaining approach allows the intense staining of the macrophage/myeloid cell markers to mask the faint CD4 expressed on these cells and to distinctly identify $\mathrm{CD} 4^{+} \mathrm{T}$ cells from myeloid lineage cells. Heat-induced epitope retrieval was performed by heating sections in $0.01 \%$ citraconic anhydride containing $0.05 \%$ Tween- 20 for TNF- $\alpha, \mathrm{Mx} 1$, and CD8 and $1 \times$ DIVA for Ki-67 and IL-17 or citrate $\mathrm{pH} 6.0$ for MPO in a pressure cooker set at $122^{\circ} \mathrm{C}$ or $125^{\circ} \mathrm{C}$ for 30 seconds. Slides were incubated with blocking buffer (TBS with $0.05 \%$ Tween-20 and $0.25 \%$ casein) for 20 minutes. For TNF- $\alpha$ and Mx1, slides were incubated for 1 hour at room temperature with mouse anti-TNF- $\alpha$ mAb (1:1,000; Abcam, catalog ab9579, clone P/T2) or with mouse anti-Mx1 mAb (1:2,000; a gift from Georg Kochs and the Department of Virology of the University of Freiburg, clone M143) diluted in blocking buffer. For all other antibodies, slides were incubated overnight at $4^{\circ} \mathrm{C}$ with rabbit anti-CD8 mAb (1:100; ThermoFisher Scientific, catalog MA1-39566, clone SP16), rabbit anti-Ki-67 mAb (1:200; ThermoFisher Scientific, catalog RM-9106, clone SP6), rabbit anti-MPO (1:5,000; Dako, catalog A0398), rabbit anti-IDO-1 (1:1,000; Millipore, catalog Ab9098), or goat anti-IL-17A (1:100; R\&D Systems, catalog AF-317-NA) diluted in blocking buffer or TBS-tween. After washing in $1 \times$ TBS with $0.05 \%$ Tween-20, endogenous peroxidases were blocked using 1.5\% (v/v) $\mathrm{H}_{2} \mathrm{O}_{2}$ in TBS, $\mathrm{pH} 7.4$, for 5 minutes, and the slides were incubated with rabbit or mouse Polink (-1 or -2) horseradish peroxidase and developed with ImmPACT DAB (3,3'-diaminobenzidine; Vector Laboratories), according to the manufacturer's recommendations. All slides were washed in tap water, counterstained with hematoxylin, mounted in Permount (Fisher Scientific), and scanned at high magnification $(\times 200)$ using the ScanScope CS or AT2 System (Aperio Technologies), yielding high-resolution data from the entire tissue section. Representative regions of interest $(500 \times 500 \mu \mathrm{m})$ were identified and high-resolution images were extracted from these whole-tissue scans. The percentage area of the positive cell zone was quantified using Photoshop CS5 and Fovea tools.

HIV-1 clade A/E lineage-specific in situ hybridization. To ensure optimal detection of productively infected cells from HIV-infected participants from Thailand, we designed a new set of HIV-1 clade A/E lineage-specific in situ hybridization riboprobes for these experiments. HIV-1 clade A/E riboprobes were generated by PCR-based cloning of target regions from the full-length infectious molecular clone pCM235 from Thailand (accession no. AF259954), provided by George Shaw (University of Pennsylvania, Philadelphia, Pennsylvania, USA). Riboprobes were generated targeting Gag (1,454-1,958; GAG F1454: 5'-CCAGTACATGCAGGGCCTAT and GAG R1958: 5'-GGCCCTTAAAATTGCCTCTC), Pol (3,998-4,570; POL F3998: 5'-ATGCGATCCATTTAGCCTTG and POL R4570: 5'-TTACTGGCCATCTTCCTGCT), the accessory genes Vif/Vpr/Vpu/Tat/Rev (5,287-5,825; VIF-REV F5782: 5'-GCAATTGGGTCATGGAGTCT and VPX R5825: 5'-CCTGGCACAATGCCTATTCT), Env (7,836-8,403; ENV F7836: 5'-GACGGTACAGGCCAGACAAT and ENV R8403: 5'-TTCCCTCTGATGATGGGAAG), and Nef (8,822-9,122; NEF F8822: 5'-GGAGGCAAGTGGTCAAAAAG and NEF R9122: 5'-TGGAGTAAATTAGCCCTTCCAGT), using primers with either phage T3 (sense) or T7 (anti-sense) promoter sequences cloned upstream of the viral sequence and pooled into a cocktail at equal concentrations. HIV-1 in situ hybridization was performed as previously described (68).

Statistics. Data in this study were analyzed with longitudinal repeated-measures ANOVA, hierarchical linear mixed-effects models $(69,70)$, standard ANOVA, regression analysis, nonparametric statistical methods, and post-hoc tests. Patients classified in different Fiebig groups (e.g., chronic, FI/II, FIII+, HIV-) were evaluated with respect to the criterion variables (e.g., Ki-67, CD4, MPO). Repeated-measures analyses (in which the "repeated" measurements are recorded for the same patient on more than one occasion) and linear mixed-effects models take into consideration the correlation/covariation of responses within the same patient over time. In addition, linear mixed-effects models account for differing variances in responses in different Fiebig groups. Following initial analyses, a posteriori (post-hoc) contrasts and follow-up, pairwise nonparametric (Wilcoxon) comparisons were performed. In all pairwise contrasts and comparisons, 
the distributions of the data in the pair of groups under consideration were evaluated with respect to their homogeneity and normality; the probability value obtained from the most appropriate model (i.e., the model that best satisfied the homogeneity of variance and normality assumptions) was selected. Compounding type I error rates for post-hoc comparisons were controlled through the use of the Benjamini-Hochberg correction (69). Probability values and adjusted probability values of less than $0.05(P<0.05)$ were considered significant, and values between 0.05 and $0.09(0.05<P<0.09)$ were considered marginally significant. Statistical analyses were performed within the R Statistical Language and Environment (71).

Study approval. The study was approved by the IRBs of Chulalongkorn University, Bangkok, Thailand, and the Walter Reed Army Institute of Research, Rockville, Maryland, USA. All participants gave informed consent.

\section{Author contributions}

$\mathrm{CD}$ designed and conducted experiments, analyzed data, and wrote the manuscript. AS designed and conducted experiments and analyzed data. LJ conducted experiments and analyzed data. XPH conducted experiments and analyzed data. DRM analyzed data. WGA performed statistical analysis. RR participated in the protocol development and data interpretation and performed GI biopsies. JLKF participated in the protocol development and data interpretation, managed cohort, and collected data. SP participated in the protocol development and data interpretation, managed cohort, and collected data. NP participated in the protocol development and managed cohort operations in Thailand. RD designed and conducted experiments and analyzed data. JMM analyzed data and wrote the manuscript. IS analyzed data and wrote the manuscript. MR participated in the protocol development, designed clinical study, and managed operation of US Military HIV Research Program studies. JHK participated in the protocol development, designed clinical study, and managed RV254 studies. TWS analyzed data and wrote the manuscript. PH analyzed data, managed US cohort, and wrote the manuscript. JDL analyzed data and wrote the manuscript. JA participated in the protocol development, designed clinical study, managed RV254 studies, coordinated research studies, and wrote the manuscript. JDE conceived, designed, and coordinated research studies; conducted experiments; analyzed data; and wrote the manuscript.

\section{Acknowledgments}

We thank our study participants and staff from the Thai Red Cross AIDS Research Centre and the Silom Community Clinic in Bangkok for their valuable contributions to this study. SEARCH is a research collaboration between the Thai Red Cross AIDS Research Centre (TRCARC), the University of Hawaii, UCSF, and the Department of Retrovirology, US Army Medical Component, Armed Forces Research Institute of Medical Sciences (Yuwadee Phuang-Ngren, Chayada Sajjawerawan, Rapee Trichavaroj, Robert O'Connell, Nantana Tantibul, Siriwat Akapirat, Surat Jongrakthaitae). The empirical component of this study was funded by the US Military HIV Research Program, Walter Reed Army Institute of Research, Rockville, Maryland, USA, under a cooperative agreement (W81XWH-07-2-0067) with the Henry M. Jackson Foundation for the Advancement of Military Medicine Inc. at the US Department of Defense. The scientific component of this study was funded in part with federal funds from the National Cancer Institute, NIH, under contract no. HHSN261200800001E, and the Delaney AIDS Research Enterprise (U19AI096109). The work of I. Sereti was supported by the intramural research program of National Institute of Allergy and Infectious Diseases/NIH. We also thank the UCLA Center for AIDS Research (CFAR) Mucosal Immunology Core Laboratory, funded by UCLA CFAR grant 5P30 AI028697, for their support and guidance regarding the isolation of mucosal mast cells. We thank the members of the Pathology Histology Laboratory of Frederick National Laboratory for Cancer Research, Leidos Biomedical Research Inc., for their service. The RV254/SEARCH 010 and RV304/SEARCH 013 study groups include Praphan Phanuphak, Nipat Teeratakulpisarn, Eugene Kroon, Donn Colby, Duanghathai Suttichom, Somprartthana Rattanamanee, Peeriya Prueksakaew, Somporn Tipsuk, Putthachard Karnsomlap, Sasiwimol Ubolyam, and Pacharin Eamyoung from SEARCH/TRCARC/HIV-NAT. See Supplemental Acknowledgments for consortia details (supplemental material available online with this article; doi:10.1172/jci.insight.87065DS1). The content of this publication does not necessarily reflect the views or policies of the US Army, the National Cancer Institute, the US Department of Defense, the Department of Health and Human Services, and the US Centers for Disease Control and Prevention nor does mention of trade names, commercial products, or organizations imply endorsement by the US Government. 
Address correspondence to: Jacob D. Estes, Frederick National Laboratory for Cancer Research, Leidos Biomedical Research Inc., 1050 Boyles Street, Bldg. 535, Rm. 411, Frederick, MD 21702, USA. Phone: 301.846.7641; E-mail: estesj@mail.nih.gov.

1. World Health Organization. HIV/AIDS: key facts. http://www.who.int/mediacentre/factsheets/fs360/en/. Updated November 2015. Accessed May 25, 2016.

2. Samji H, et al. Closing the gap: increases in life expectancy among treated HIV-positive individuals in the United States and Canada. PLoS ONE. 2013;8(12):e81355.

3. Wada N, Jacobson LP, Cohen M, French A, Phair J, Muñoz A. Cause-specific life expectancies after 35 years of age for human immunodeficiency syndrome-infected and human immunodeficiency syndrome-negative individuals followed simultaneously in long-term cohort studies, 1984-2008. Am J Epidemiol. 2013;177(2):116-125.

4. Deeks SG, Phillips AN. HIV infection, antiretroviral treatment, ageing, and non-AIDS related morbidity. BMJ. 2009;338:a3172.

5. Lohse N, et al. Survival of persons with and without HIV infection in Denmark, 1995-2005. Ann Intern Med. 2007;146(2):87-95.

6. Somsouk M, et al. Gut epithelial barrier and systemic inflammation during chronic HIV infection. AIDS. 2015;29(1):43-51.

7. Brenchley JM, et al. Microbial translocation is a cause of systemic immune activation in chronic HIV infection. Nat Med. 2006;12(12):1365-1371.

8. Ancuta P, et al. Microbial translocation is associated with increased monocyte activation and dementia in AIDS patients. PLoS ONE. 2008;3(6):e2516.

9. Gupta SK, et al Markers of renal disease function are associated with systemic inflammation in HIV infection. HIV Med. 2015;16(10):591-598.

10. Estes JD, et al. Antifibrotic therapy in simian immunodeficiency virus infection preserves CD4+ T-cell populations and improves immune reconstitution with antiretroviral therapy. J Infect Dis. 2015;211(5):744-754.

11. Hsu DC, Sereti I, Ananworanich J. Serious Non-AIDS events: immunopathogenesis and interventional strategies. AIDS Res Ther. 2013;10(1):29.

12. Dunham RM, et al. Discordance between peripheral and colonic markers of inflammation during suppressive ART. $J$ Acquir Immune Defic Syndr. 2014;65(2):133-141.

13. Ipp H, Zemlin AE, Erasmus RT, Glashoff RH. Role of inflammation in HIV-1 disease progression and prognosis. Crit Rev Clin Lab Sci. 2014;51(2):98-111.

14. Guadalupe $\mathrm{M}$, et al. Severe $\mathrm{CD} 4+\mathrm{T}$-cell depletion in gut lymphoid tissue during primary human immunodeficiency virus type 1 infection and substantial delay in restoration following highly active antiretroviral therapy. J Virol. 2003;77(21):11708-11717.

15. Brenchley JM, et al. CD4+ T cell depletion during all stages of HIV disease occurs predominantly in the gastrointestinal tract. J Exp Med. 2004;200(6):749-759.

16. Costiniuk CT, Angel JB. Human immunodeficiency virus and the gastrointestinal immune system: does highly active antiretroviral therapy restore gut immunity? Mucosal Immunol. 2012;5(6):596-604.

17. Eisele E, Siliciano RF. Redefining the viral reservoirs that prevent HIV-1 eradication. Immunity. 2012;37(3):377-388

18. Okoye A, et al. Progressive CD4+ central memory T cell decline results in CD4+ effector memory insufficiency and overt disease in chronic SIV infection. J Exp Med. 2007;204(9):2171-2185.

19. Moir S, et al. B cells in early and chronic HIV infection: evidence for preservation of immune function associated with early initiation of antiretroviral therapy. Blood. 2010;116(25):5571-5579.

20. Buzon MJ, et al. Long-term antiretroviral treatment initiated at primary HIV-1 infection affects the size, composition, and decay kinetics of the reservoir of HIV-1-infected CD4 T cells. J Virol. 2014;88(17):10056-10065.

21. Hey-Cunningham WJ, et al. Early antiretroviral therapy with raltegravir generates sustained reductions in HIV reservoirs but not lower T-cell activation levels. AIDS. 2015;29(8):911-919.

22. Chéret $A$, et al. Intensive five-drug antiretroviral therapy regimen versus standard triple-drug therapy during primary HIV-1 infection (OPTIPRIM-ANRS 147): a randomised, open-label, phase 3 trial. Lancet Infect Dis. 2015;15(4):387-396.

23. Le T, et al. Enhanced CD4+ T-cell recovery with earlier HIV-1 antiretroviral therapy. $N$ Engl J Med. 2013;368(3):218-230.

24. Hocqueloux L, et al. Long-term antiretroviral therapy initiated during primary HIV-1 infection is key to achieving both low HIV reservoirs and normal T cell counts. J Antimicrob Chemother. 2013;68(5):1169-1178.

25. Schuetz A, et al. Initiation of ART during early acute HIV infection preserves mucosal Th17 function and reverses HIV-related immune activation. PLoS Pathog. 2014;10(12):e1004543.

26. Kijak GH, et al. Molecular evolution of the HIV-1 Thai epidemic between the time of RV144 immunogen selection to the execution of the vaccine efficacy trial. $J$ Virol. 2013;87(13):7265-7281.

27. Oyomopito RA, et al. Evaluating immunologic response and clinical deterioration in treatment-naive patients initiating first-line therapies infected with HIV-1 CRF01_AE and subtype B. J Acquir Immune Defic Syndr. 2013;62(3):293-300.

28. Fiebig EW, et al. Dynamics of HIV viremia and antibody seroconversion in plasma donors: implications for diagnosis and staging of primary HIV infection. AIDS. 2003;17(13):1871-1879.

29. Estes JD, et al. Damaged intestinal epithelial integrity linked to microbial translocation in pathogenic simian immunodeficiency virus infections. PLoS Pathog. 2010;6(8):e1001052.

30. Sereti I, et al. Decreases in colonic and systemic inflammation in chronic HIV infection after IL-7 administration. PLoS Pathog. 2014;10(1):e1003890.

31. Heise C, Miller CJ, Lackner A, Dandekar S. Primary acute simian immunodeficiency virus infection of intestinal lymphoid tissue is associated with gastrointestinal dysfunction. J Infect Dis. 1994;169(5):1116-1120.

32. Li Q, et al. Simian immunodeficiency virus-induced intestinal cell apoptosis is the underlying mechanism of the regenerative enteropathy of early infection. J Infect Dis. 2008;197(3):420-429

33. Sankaran S, et al. Rapid onset of intestinal epithelial barrier dysfunction in primary human immunodeficiency virus infection is 
driven by an imbalance between immune response and mucosal repair and regeneration. $J$ Virol. 2008;82(1):538-545.

34. Baroncelli S, et al. Microbial translocation is associated with residual viral replication in HAART-treated HIV+ subjects with $<50$ copies/ml HIV-1 RNA. J Clin Virol. 2009;46(4):367-370.

35. Jiang W, et al. Plasma levels of bacterial DNA correlate with immune activation and the magnitude of immune restoration in persons with antiretroviral-treated HIV infection. J Infect Dis. 2009;199(8):1177-1185.

36. Ananworanich J, et al. Impact of multi-targeted antiretroviral treatment on gut $\mathrm{T}$ cell depletion and HIV reservoir seeding during acute HIV infection. PLoS ONE. 2012;7(3):e33948.

37. Ananworanich J, et al. Virological and immunological characteristics of HIV-infected individuals at the earliest stage of infection. J Virus Erad. 2016;2:43-48.

38. Massanella M, Fromentin R, Chomont N. Residual inflammation and viral reservoirs: alliance against an HIV cure. Curr Opin HIV AIDS. 2016;11(2):234-241.

39. Mehandru S, et al. Neutralization profiles of newly transmitted human immunodeficiency virus type 1 by monoclonal antibodies 2G12, 2F5, and 4E10. J Virol. 2004;78(24):14039-14042.

40. Mehandru S, et al. Lack of mucosal immune reconstitution during prolonged treatment of acute and early HIV-1 infection. PLoS Med. 2006;3(12):e484.

41. Guadalupe M, et al. Viral suppression and immune restoration in the gastrointestinal mucosa of human immunodeficiency virus type 1-infected patients initiating therapy during primary or chronic infection. $J$ Virol. 2006;80(16):8236-8247.

42. Ciccone EJ, et al. Cycling of gut mucosal CD4+ T cells decreases after prolonged anti-retroviral therapy and is associated with plasma LPS levels. Mucosal Immunol. 2010;3(2):172-181.

43. Karris MY, et al. Predictors of virologic response in persons who start antiretroviral therapy during recent HIV infection. AIDS. 2014;28(6):841-849.

44. Pelletier M, et al. Evidence for a cross-talk between human neutrophils and Th17 cells. Blood. 2010;115(2):335-343.

45. Dandekar S, George MD, Bäumler AJ. Th17 cells, HIV and the gut mucosal barrier. Curr Opin HIV AIDS. 2010;5(2):173-178.

46. Favre D, et al. Tryptophan catabolism by indoleamine 2,3-dioxygenase 1 alters the balance of TH17 to regulatory T cells in HIV disease. Sci Transl Med. 2010;2(32):32ra36.

47. Chege D, et al. Sigmoid Th17 populations, the HIV latent reservoir, and microbial translocation in men on long-term antiretroviral therapy. AIDS. 2011;25(6):741-749.

48. Brenchley JM, et al. Differential Th17 CD4 T-cell depletion in pathogenic and nonpathogenic lentiviral infections. Blood. 2008;112(7):2826-2835.

49. Salgado M, Rallón NI, Rodés B, López M, Soriano V, Benito JM. Long-term non-progressors display a greater number of Th17 cells than HIV-infected typical progressors. Clin Immunol. 2011;139(2):110-114.

50. Klatt NR, et al. Loss of mucosal CD103+ DCs and IL-17+ and IL-22+ lymphocytes is associated with mucosal damage in SIV infection. Mucosal Immunol. 2012;5(6):646-657.

51. Kök A, et al. Early initiation of combined antiretroviral therapy preserves immune function in the gut of HIV-infected patients. Mucosal Immunol. 2015;8(1):127-140.

52. Sabat R, Witte E, Witte K, Wolk K. IL-22 and IL-17: an overview. In Quesniaux V, Ryffel B, Di Padova F, eds. IL-17, IL-22 and Their Producing Cells: Role in Inflammation and Autoimmunity. Basel, Switzerland: Springer; 2013:11-36.

53. Estes J, et al. Collagen deposition limits immune reconstitution in the gut. J Infect Dis. 2008;198(4):456-464.

54. Mavigner M, et al. Altered CD4+ T cell homing to the gut impairs mucosal immune reconstitution in treated HIV-infected individuals. J Clin Invest. 2012;122(1):62-69.

55. Gay CL, et al. Efficacy of NNRTI-based antiretroviral therapy initiated during acute HIV infection. AIDS. 2011;25(7):941-949.

56. Jain V, et al. Antiretroviral therapy initiated within 6 months of HIV infection is associated with lower T-cell activation and smaller HIV reservoir size. J Infect Dis. 2013;208(8):1202-1211.

57. Burdo TH, et al. Soluble CD163, a novel marker of activated macrophages, is elevated and associated with noncalcified coronary plaque in HIV-infected patients. J Infect Dis. 2011;204(8):1227-1236.

58. Burdo TH, Weiffenbach A, Woods SP, Letendre S, Ellis RJ, Williams KC. Elevated sCD163 in plasma but not cerebrospinal fluid is a marker of neurocognitive impairment in HIV infection. AIDS. 2013;27(9):1387-1395.

59. Fahey JL, et al. The prognostic value of cellular and serologic markers in infection with human immunodeficiency virus type 1 . N Engl J Med. 1990;322(3):166-172.

60. Hazenberg MD, et al. Persistent immune activation in HIV-1 infection is associated with progression to AIDS. AIDS. 2003;17(13):1881-1888.

61. Schellekens PT, Koot M, Roos MT, Tersmette M, Miedema F. Immunologic and virologic markers determining progression to AIDS. J Acquir Immune Defic Syndr Hum Retrovirol. 1995;10 Supp1 2:S62-S66.

62. Lawn SD, Butera ST, Folks TM. Contribution of immune activation to the pathogenesis and transmission of human immunodeficiency virus type 1 infection. Clin Microbiol Rev. 2001;14(4):753-777

63. Hunt PW, et al. Impact of CD8+ T-cell activation on CD4+ T-cell recovery and mortality in HIV-infected Ugandans initiating antiretroviral therapy. AIDS. 2011;25(17):2123-2131.

64. Ho JE, et al. The association of CD4+ T-cell counts and cardiovascular risk in treated HIV disease. AIDS. 2012;26(9):11151120 .

65. Ananworanich J, et al. Incidence and characterization of acute HIV-1 infection in a high-risk Thai population. J Acquir Immune Defic Syndr. 2008;49(2):151-155.

66. Estes JD, et al. Early resolution of acute immune activation and induction of PD-1 in SIV-infected sooty mangabeys distinguishes nonpathogenic from pathogenic infection in rhesus macaques. J Immunol. 2008;180(10):6798-6807.

67. Hao XP, et al. Experimental colitis in SIV-uninfected rhesus macaques recapitulates important features of pathogenic SIV infection. Nat Commun. 2015;6:8020.

68. Brenchley JM, et al. Differential infection patterns of CD4+ T cells and lymphoid tissue viral burden distinguish progressive and nonprogressive lentiviral infections. Blood. 2012;120(20):4172-4181.

69. Klipper-Aurbach Y, et al. Mathematical formulae for the prediction of the residual beta cell function during the first two years 
of disease in children and adolescents with insulin-dependent diabetes mellitus. Med Hypotheses. 1995;45(5):486-490.

70. Gong J, Pinheiro JC, DeMets DL. Estimating significance level and power comparisons for testing multiple endpoints in clinical trials. Control Clin Trials. 2000;21(4):313-329.

71. R Development Core Team. R: A Language and Environment for Statistical Computing. Vienna, Austria: R Foundation for Statistical Computing; 2011 